\title{
An Assessment of Mixed-Modeling Approaches for Characterizing Profiles of Time-Varying Response and Predictor Variables
}

\author{
L. V. Madden and P. A. Paul
}

Department of Plant Pathology, Ohio State University, Wooster 44691.

Accepted for publication 4 June 2010.

\begin{abstract}
Madden, L. V., and Paul, P. A. 2010. An assessment of mixed-modeling approaches for characterizing profiles of time-varying response and predictor variables. Phytopathology 100:1015-1029.

A general statistical modeling approach was tested for characterizing the relationship between pathogen inoculum density (or other biological response variables) and environmental variables when the data are collected as temporal profiles of observations within multiple locations or years. The approach, based on the use of linear mixed models, simultaneously accounts for serial correlations of the observations within each time profile, the random effects of location-year (or other grouping factors), and the cross-correlation of the environmental variables, and is appropriate when the environmental effects on the response variable or its transformation $(Y)$ are distributed over several times (e.g., days). Stability and precision of parameter estimates for environmental effects over multiple time lags were achieved through the use of polynomial constraints within a likelihood-based full mixed-model fit; from the parameter estimates, marginal effects of environmental variables and weights for individual time lags were determined. The mixed model was directly

unrelated to the environmental variables being considered. The new approach described here (with or without a smoothing function) generalizes a previously used-and computationally less demanding-two-stage (composite) approach. In the previous approach, constrained parameter estimates and associated weights were first determined without consideration of serial correlation, cross-correlation of environmental variables, and the random effects of location-year; then, a mixed-model fit was accomplished using the fixed time-lag weights derived in the first step. Using data for inoculum density of Gibberella zeae on wheat spikes from 27 location-years, similar results were achieved with the full mixed model and the two-stage approaches, in terms of both the calculated parameters and predictions of $Y$. With the use of smoothing functions, the precision of the predictions was improved but the general conclusions regarding environmental effects on $Y$ were not affected. Thus, in the particular example data set, previously derived conclusions regarding environmental effects on inoculum density were robust in terms of the statistical methodology used in analysis; most researchers will find the two-stage approach much easier to implement for the analysis of multiple profiles of time-varying observations.
\end{abstract} expanded, through the incorporation of smoothing functions, to potentially account for possible longer-term trends in the temporal profiles

The probability of plant infection by a fungal pathogen at any moment during an epidemic is a function of inoculum (e.g., spore) density, environmental favorability, and host susceptibility (29). Depending on the pathosystem, spores can be produced on plants infected during the current epidemic (for polycyclic diseases), on living parts of other plant species, or on dead plant debris. The inoculum source may be either local or more distant, and spore production and dispersal themselves are functions of the environment.

Profiles of inoculum density are commonly graphed to characterize the temporal (e.g., daily) fluctuations of spores in the air or on plant surfaces and to determine the variation in density among years, locations, or treatments $(13,14,22,25)$. Similar profiles are commonly prepared for density of human allergens $(2,9,16)$ and other biological response variables. A characteristic of these profiles is the high variation in density among the time points (which can be attenuated using a log transformation), coupled with general temporal trends where peaks and valleys can be seen. For quantification of disease risk, it would be valuable to be able to predict inoculum density or other response variables based on environmental (and other) variables. Perhaps surprisingly, although many authors have detected significant correlations of weather variables with inoculum, satisfactory prediction has been difficult

Corresponding author: L. V. Madden; E-mail address: madden.1@ osu.edu

doi:10.1094/PHYTO-01-10-0001

(c) 2010 The American Phytopathological Society
Additional keywords: maximum likelihood, radial smoothing.

to accomplish. This is because the effect of any weather variable likely is distributed over multiple days, weather variables can have high serial (auto-) correlations over time, and spore density is influenced by more than just weather variables (13). Several methods have been proposed to quantify the interrelationships of time-dependent biological response variables and environmental variables $(3,11,34,35,40,41,46,47)$, especially when there are long profiles of observations. Most of these methods have been developed for or adopted in human epidemiology, especially for characterizing human mortality in relation to air pollutants.

Polynomial distributed lag (PDL) regression is an especially useful method for relating a temporally varying response variable to one or more temporally varying predictor variables $(1,4,17,39)$. The advantages of PDL regression analysis are that (i) it can greatly reduce the effect of serial correlation of the predictor variables on the statistical results (giving a more stable set of parameter estimates) and (ii) it provides a formal protocol for determining the length of the time window where predictor variables significantly affect the response variable, and the weights for the predictors on each day of the window $(39,43)$. These advantages are achieved by constraining the model parameters (not the variables in the model) to follow a polynomial of an order (power) suggested by the data (17). Versions of PDL regression for single (long) profiles of observations have been used in several studies $(3,9,23,43,44)$ but the methodology has had only limited use in plant pathology $(23,33)$.

Most single profiles of inoculum density over time (e.g., daily spore densities in a spore sampler or on a plant surface) will not 
be long enough for characterizing the functional relationship between inoculum and weather (33), especially if the effect of the weather variable is distributed over a long time window. However, combining the data from several profiles of observations (e.g., from different locations and years) can lead to stable modelfitting results, if one can assume that the underlying functional relationship between the response variable and the weather variable does not vary among profiles. This latter approach, however, requires the use of mixed models $(26,38)$ to account for random effects of the grouping variables (e.g., years or locations), intercorrelations (i.e., cross-correlations) of the predictors, and the serial correlations of residuals within the groups on the results. Unfortunately, PDL constraints are not directly or automatically implemented within standard mixed-model fitting programs. Therefore, a two-stage process can be used as an approximation, in which a PDL regression analysis is first conducted separately for each predictor variable using the pooled data, ignoring the serial correlation of the residuals, cross-correlation of predictor variables, and any random effects (33). Both the length of the time window and the polynomial order are determined in this first stage. Composite weather variables are then constructed based on the estimated weights found for each predictor in the PDL analysis. In the second stage, a linear mixed model $(26,38)$ is fitted to the response variable in relation to multiple composite weather variables while simultaneously accounting for any random effects and serial- or cross-correlations that are manifested when composite predictor variables are utilized. Paul et al. (33) recently took this two-stage approach to characterize the relationship between the log of inoculum density of Gibberella zeae (Fusarium graminearum) on wheat spikes over time and several different weather variables, using profiles of observations from 27 location-years in the United States.

This two-stage analysis is an approximation of more general approaches (see below) for analysis of multiple profiles of observations that were not originally attempted by Paul et al. (33) because of the high computational demands of these alternatives. In particular, by constructing and using composite predictors over specified time windows, the functional relationship between the response and each predictor variable (i.e., the weights given to the predictors on each day of the window) is not allowed to vary in the mixed model. Therefore, the cross-correlations of the different predictors, serial correlations, and the random effects in the models are not allowed to affect the weights given to the lagged weather variables. Accordingly, the model fits may not be optimum for prediction or for estimation of parameters. Moreover, if systematic or general changes in the response variable over time are related to predictor variables not considered in the analysis, biased parameter estimates could be obtained $(6,34,39,48)$.

The overall objective of the current study was to develop and evaluate linear mixed models for temporal profiles of a response variable (e.g., spore density) that allowed the predictor-variable weights (for appropriate time windows) to be estimated concurrently with other terms in the models while simultaneously accounting for cross-correlations of predictor variables, random effects, and serial correlations of residuals. A likelihood approach was taken for model fitting $(26,38)$, and model performance (including estimates of the probability of a given model being superior to others) was assessed through likelihood-based statistics (7). A direct comparison of results with those obtained with the two-stage approach is made. The models were then expanded to include smoothing splines $(28,36,45)$ that could potentially account for general trends in spore density not accounted for by other model terms and, thus, lead to less biased estimates of weather-variable parameters. The likelihood-based protocols developed herein can be applied to any time-varying and possibly serially correlated variable with data collected from multiple locations or years.

\section{THEORY AND APPROACHES}

Example data set. The developed models (explained below) were fitted to the data presented by Paul et al. (33) on the inoculum density of the Fusarium head blight (FHB) pathogen on wheat spikes. Briefly, wheat spikes were sampled in 1999-2005 in plots in five U.S. states and one Canadian province, although data were not available in all years at all locations. In total, 27 location-years were represented in the data set, including 4 years from Indiana, 6 from North Dakota, 7 from Ohio, 4 from Pennsylvania, 5 from South Dakota, and 1 from Manitoba (Canada).

Five spikes from each of several research plots were collected daily between Feekes growth stage 10 and 11.2 (29) and washed with distilled water, and aliquots of wash suspensions were transferred to selective medium. Colonies on medium were grown for 7 to 10 days at room temperature and CFU per plate were determined. From these numbers, the CFU of G. zeae per spike per day, averaged across all sampled wheat plots and spikes per plot, were determined.

Temperature, relative humidity, rainfall, wind speed, and wind direction were measured electronically in each location-year. Several weather variables were constructed to summarize the daily weather conditions. These included averages (for the entire day or for daytime and nighttime periods) and number of hours when certain conditions were met, such as hours with temperature $>9^{\circ} \mathrm{C}$ or relative humidity $>90 \%$ (33). Armstrong (3) took an analogous approach in constructing temperature variables in an analysis of time-varying human mortality.

Paul et al. (33) found several weather variables significantly related to $\log$ of daily spores per wheat spike. Most of these variables were intercorrelated because they consisted of the same original measurements expressed in various forms. Six variables were selected for additional analysis and, from these, five different linear mixed models were developed with either two or three predictors. The analysis in the current investigation was restricted to the same six weather variables. The six variables are average daily relative humidity of the air (AVARH), average daytime (0600 to $1800 \mathrm{~h}$ ) relative humidity of the air (AVDARH), average daytime air temperature $\left({ }^{\circ} \mathrm{C}\right)$ (AVDAT), number of hours per day with air temperature of 15 to $30^{\circ} \mathrm{C}$ (AT1530), number of hours per day with air temperature $\geq 15^{\circ} \mathrm{C}$ and relative humidity $\geq 90 \%$ (AT15RH90), and daily average rainfall intensity $(\mathrm{mm} / \mathrm{h}$ ) (INTENSITY).

Model notation. We define $Y$ as the response variable and $t$ as time (e.g., days); $Y_{t}$ as the response variable on day $t$. For the FHB data set in particular, we let $C_{t}$ represent the number of CFU of $G$. zeae per spike on day $t$ in a given location and year. The current analysis is based on the log-transformation of these numbers; thus, $Y_{t}=\ln \left(C_{t}+1\right)$. For multiple groups of $Y_{t}$ profiles, we designate the individual group with the $i$ subscript; an additional level of grouping can be represented by the $j$ subscript. For the FHB data, we specify, as needed, the location by $i$ and the year within location $i$ by $j$.

A generic predictor variable on day $t$ is represented by $X_{t}$; a predictor weather variable on a previous day is given by $X_{t-m}$, where $m$ is the temporal lag. Although predictor variables can involve more than weather or environment, for ease of presentation, we often refer to $X_{t}$ (or $X_{t-m}$ ) as a weather variable. The maximum temporal lag is $n(m=0, \ldots, n)$. For instance, the value of the weather variable 2 days before day $t(m=2)$ is written as $X_{t-2}$. Superscripts are used to denote different weather variables. For instance, $X_{t}^{(1)}$ denotes the first weather variable for day $t$. Parameters corresponding to specific temporal lags are designated with $m$ subscripts (e.g., $\beta_{m}$ for lag $m$ ), whereas parameters corresponding to specific weather variables are designated with superscripts (e.g., $\beta_{m}^{(1)}$ for the lag $m$ and the first weather variable).

Polynomial distributed lag models. We repeat some of the model formulations used in Paul et al. (33) for the two-stage 
analysis so that expansions and generalizations can be clearly seen, and to facilitate direct comparisons of results. For any given profile, we assume that $Y_{t}$ is affected by weather variables on the current day and on the previous $n$ days in a linear manner. The time period from day 0 to day $n$ is known as the time window. The model for a single weather variable can be written generally as:

$$
Y_{t}=\alpha+\beta_{0} X_{t}+\beta_{1} X_{t-1}+\beta_{2} X_{t-2}+\ldots+\beta_{\mathrm{n}} X_{t-n}+e_{t}
$$

in which $\beta_{0}-\beta_{\mathrm{n}}$ are parameters corresponding to the predictor variable on the current and previous $n$ days; each $\beta_{\mathrm{m}}$ parameter (e.g., $\beta_{2}$ ) represents the influence given to the $X$ value for a particular temporal lag; $\alpha$ is a parameter representing the expected value of $Y_{t}$ when all the $X \mathrm{~s}$ are 0 . The term $e_{t}$ is the residual (unexplained-variation term) for day $t$. Equation 1a can be written succinctly as:

$$
Y_{t}=\alpha+\sum_{m=0}^{n} \beta_{m} X_{t-m}+e_{t}
$$

There are $n+2$ parameters in equation $1 \mathrm{~b}\left(\alpha, \beta_{0}-\beta_{\mathrm{n}}\right)$. Because the lagged $X_{t}$ variables will be, in general, highly correlated, use of unconstrained ordinary least squares for parameter estimation will result in very imprecise estimates of the parameters and an unstable model fit $(1,17,39)$. Several approaches are possible for diminishing the impact of the correlations in the model fit $(3,11$, $34,40,41,47)$. These approaches typically constrain the parameter estimates either implicitly or explicitly. One powerful approach to the problem is PDL model fitting $(1,17,20)$.

For a PDL, the $\beta_{m}$ parameters are constrained to fall on a polynomial curve of degree $d(1,32)$. A common way of expressing this succinctly (if one adopts a special mathematical construct of $\left.0^{0} \equiv 1\right)$ is with the expression (39):

$$
\beta_{m}=\sum_{k=0}^{d} \eta_{k} m^{k}
$$

in which $k$ is an index to designate a particular power in a polynomial function of degree $d(k=0, \ldots, d)$, and the $\eta_{k}$ s are new parameters $\left(\eta_{0}-\eta_{d}\right)$. For instance, with a quadratic polynomial $(d=2)$, there are three $\eta$ parameters, $\eta_{0}, \eta_{1}$, and $\eta_{2}$ (corresponding to $k=0,1$, and 2). The original $\beta_{m}$ parameters can be written as: $\beta_{0}=\eta_{0}, \beta_{1}=\eta_{0}+\eta_{1}+\eta_{2}$, and, in general, $\beta_{m}=\eta_{0}+$ $m \eta_{1}+m^{2} \eta_{2}$.

As shown in the Appendix, with the constraints in equation 2, equation $1 \mathrm{~b}$ can be written in terms of $d+1$ constructed variables as:

$$
Y_{t}=\alpha+\sum_{k=0}^{d} \eta_{k} Z_{(k) t}+e_{t}
$$

in which $Z_{(k) t}$ represents a linear combination of the lagged weather variable. With $d=2$, there are three $Z_{(k) t}$ terms $(k=0,1$, 2 ); in general, for power $k$,

$$
Z_{(k) t}=\sum_{m=0}^{n} m^{k} X_{t-m}
$$

Equation 3, with $d+2$ parameters (i.e., $\alpha, \eta_{0}-\eta_{d}$ ), provides a more parsimonious (although not necessarily a more accurate) description of the data than does equation $1 \mathrm{~b}(n+2$ parameters) as long as $d<n$. The lower the value of the polynomial order, the simpler the model relative to the unconstrained model. If equation 3 is used, then the original $\beta_{m}$ parameters of equation $1 \mathrm{~b}$ are obtained from the $\eta_{k}$ parameters by using equation 2 .

Composite model. With equation 1a (or $1 b$ ), the sum of the $\beta_{m}$ parameters $\left(\beta^{*}=\Sigma \beta_{m}\right)$ is the marginal effect of the predictor variable on $Y_{t}$. Dividing each $\beta_{m}$ value by $\beta^{*}$ produces the weight given to the predictor variable at temporal lag $m$ in predicting $Y$. That is, we define $w_{m}=\beta_{m} / \beta^{*}$, which means that the sum of the $w_{m}$ values equals 1 , by definition. If the $\beta_{m}$ values were con- strained to fall on a polynomial (equation 2) (in other words, if the $\beta_{m}$ parameters were obtained from the $\eta_{k}$ parameters), then the $w_{m}$ weights would also fall on a polynomial. Using the weights, equation $1 \mathrm{~b}$ can then be written as:

$$
Y_{t}=\alpha+\beta^{*} \sum_{m=0}^{n} w_{m} X_{t-m}+e_{t}
$$

We can further define

$$
X_{t}^{*}=\sum_{m=0}^{n} w_{m} X_{t-m}
$$

as a so-called composite variable, and rewrite equation 4a as:

$$
Y_{t}=\alpha+\beta^{*} X_{t}^{*}+e_{t}
$$

If the weights were known (or assumed known), then $\beta^{*}$ could be estimated with ordinary regression analysis (for a single locationyear) because $X_{t}^{*}$ in equation $4 \mathrm{~b}$ involves no unknowns.

Multiple predictors. The distributed-lag model of equation $1 \mathrm{~b}$ can be easily expanded for multiple predictor variables. For instance, if there were three separate weather variables, the model can be written as:

$$
Y_{t}=\alpha+\sum_{m=0}^{n} \beta_{m}^{(1)} X_{t-m}^{(1)}+\sum_{m=0}^{n} \beta_{m}^{(2)} X_{t-m}^{(2)}+\sum_{m=0}^{n} \beta_{m}^{(3)} X_{t-m}^{(3)}+e_{t}
$$

where the superscript identifies a weather variable and the corresponding parameters. The maximum temporal lag can also depend on the predictor variable (e.g., $n^{(2)}$ for the second predictor variable). To represent this in equation $6 \mathrm{a}, n$ in the summations would be replaced by $n^{(1)}, n^{(2)}$, and $n^{(3)}$, respectively, for predictors 1,2 , and 3. One can sum the parameters for each predictor (e.g., $\left.\beta^{(2)^{*}}=\sum \beta_{m}^{(2)}\right)$ to obtain the marginal effect of the predictor in predicting $Y_{t}$, and determine the weights given to each temporal lag for each predictor (e.g., $\left.w_{m}^{(2)}=\beta_{m}^{(2)} / \beta^{(2)^{*}}\right)$. Equation $6 \mathrm{a}$ can then be written as:

$$
Y_{t}=\alpha+\beta^{(1)^{*}} \sum_{m=0}^{n} w_{m}^{(1)} X_{t-m}^{(1)}+\beta^{(2)^{*}} \sum_{m=0}^{n} w_{m}^{(2)} X_{t-m}^{(2)}+\beta^{(3)^{*}} \sum_{m=0}^{n} w_{m}^{(3)} X_{t-m}^{(3)}+e_{t}
$$

By applying equation 5 to each of the predictors (with different weights for each), equation $6 \mathrm{~b}$ can also be written succinctly as:

$$
Y_{t}=\alpha+\beta^{(1)^{*}} X_{t}^{(1)^{*}}+\beta^{(2)^{*}} X_{t}^{(2)^{*}}+\beta^{(3)^{*}} X_{t}^{(3)^{*}}+e_{t}
$$

Furthermore, equation $6 \mathrm{a}$ can be reduced to a PDL as:

$$
Y_{t}=\alpha+\sum_{k=0}^{d} \eta_{k}^{(1)} Z_{(k) t}^{(1)}+\sum_{k=0}^{d} \eta_{k}^{(2)} Z_{(k) t}^{(2)}+\sum_{k=0}^{d} \eta_{k}^{(3)} Z_{(k) t}^{(3)}+e_{t}
$$

where, as above, the superscripts refer to the different predictors and their corresponding parameters, and the $Z_{(k) t}$ predictor variables are linear combinations of the $X_{t-m}$ variables (see Appendix). Moreover, the polynomial power can be different for each predictor (e.g., $d^{(2)}$ for the second predictor); with different degrees (as usual), the $d$ in the summations of equation 7 would simply be replaced by $d^{(1)}, d^{(2)}$, and $d^{(3)}$ for predictors 1,2 , and 3 , respectively. The estimates of the $\beta_{m}$ parameters (or the weights, $w_{m}$ ) are recovered from the parameter estimates of equation 7 using the polynomial function in equation 2 .

Mixed models. Equations 6a, 6c, and 7 can all be expanded to mixed models to account for the effects of groups (such as location and year within location) or other random effects on $Y_{t}$, and also to account for the serial correlation of residuals within location-years (21). The inclusion of multiple predictors in the same model also accounts for the cross-correlation of the predictors. For the case of multiple locations and years, one generalization of equation $6 \mathrm{c}$ is given as:

$Y_{i j t}=\alpha+\beta^{(1)^{*}} X_{i j t}^{(1)^{*}}+\beta^{(2)^{*}} X_{i j t}^{(2)^{*}}+\beta^{(3)^{*}} X_{i j t}^{(3)^{*}}+L_{i}+T(L)_{i j}+e_{i j t}$ 
in which $i j t$ subscript represents the $t$ th day of the $j$ th year within the $i$ th location. $L_{i}$ is the effect of the $i$ th location on the response (considered a fixed effect here, but could be considered a random effect), and $T(L)_{i j}$ is the effect of the $j$ th year of the $i$ th location on the response (considered a random effect, with a single variance component $\left.\left[\sigma_{T(L)}^{2}\right]\right)$. It is further assumed that the residuals $\left(e_{i j t}\right)$ are correlated because data are collected over time within groups. Note that inclusion of $T(L)_{i j}$ as a random term assures that observations (or residuals) within a location-year will be correlated (as long as the corresponding variance estimate for this random effect is not zero) but this correlation will not depend on the temporal difference of the observations; incorporating a dependence structure to $e_{i j t}$ adds an additional level of within-locationyear correlation that depends on how far apart the observations are (26). Different versions of equation 8 were successfully used by Paul et al. (33) for characterizing FHB inoculum density over time. However, fitting of equation 8 is a two-stage process, where the composite predictor variables in equation 8 (e.g., $X_{i j t}^{(1)^{*}}$; see equation 5) are obtained from weights obtained separately for each predictor using a PDL regression analysis for individual predictors. That is, PDL regression analysis is conducted in the first stage to obtain the weights, and then equation 8 is directly fitted to the data in the second stage using a mixed-model-based method. The derived weights from the first stage-which cannot change in the second stage of analysis-are obtained without consideration of the cross-correlation of the different predictor variables, any random effects, or the serial correlation of the residuals.

Direct fitting of a mixed-model version of equation 6a (with no constraints on the $\beta_{m}$ parameters) is unrealistic for the same reasons that fitting of equation $1 \mathrm{~b}$ is unrealistic for a single profile of observations; that is, the solution would be unstable because of the imprecision and likely high correlation of the unconstrained parameter estimates (39). Instead, the following mixed-model version of equation 7 can be fitted:

$Y_{i j t}=\alpha+\sum_{k=0}^{d} \eta_{k}^{(1)} Z_{(k) i j t}^{(1)}+\sum_{k=0}^{d} \eta_{k}^{(2)} Z_{(k) j i t}^{(2)}+\sum_{k=0}^{d} \eta_{k}^{(3)} Z_{(k) i j t}^{(3)}+L_{i}+T(L)_{i j}+e_{i j t}$

Equation 9 is a special case of a general constrained mixed model discussed by Edwards (12). For a chosen maximum time lag $(n)$ and polynomial degree $(d)$, the $Z_{(k)}$ variables are constructed and used as the predictor variables in equation 9 (see the Appendix for details on the $Z_{(k)}$ variables). After estimating the $\eta_{k}$ parameters, the underlying $\beta_{m}$ parameters for each predictor variable can be estimated using equation 2. Furthermore, the marginal-effect parameter $\left(\beta^{*}\right)$ can be estimated by summing the $\beta_{m}$ estimates for each predictor.

For convenience of expression within the text that follows, the $t$ or the ijt subscripts for the response and predictor variables may be suppressed when the meaning is clear by context. Moreover, the superscripts for the different predictors or corresponding parameters may also be suppressed when the meaning is clear.

Radial smoothing. The preceding models were based on the premise that the expected response variable (e.g., expected log of the daily spore count) in a given location and year systematically varies with selected weather (predictor) variables, with the effect spread out over $m$ days (the time window). However, the observed pattern of $Y$ versus time may also depend on the numerous other variables not measured, such as longer-term climatic conditions locally and more distantly (where some of the spores may have originated), physiological status of the crop residue where inoculum is produced, and so on. More specifically, temporal patterns to $Y$ unrelated to the utilized $X$ predictors can lead to biased $\beta_{m}$ parameter estimates $(36,39)$, especially if there are systematic patterns to the other (unrecorded) variables. One approach to dealing with this situation, which is usually taken in human epidemiological studies, is to empirically adjust for (or correct for) longer-term or large-scale temporal patterns of the response variable so that shorter-term (or more immediate) effects of the predictor variables of interest can be estimated with less bias $(6,34,39,46)$. Because the factors affecting $Y$, other than the observed weather variables considered here, are not defined, there is no a priori defined temporal pattern to the profiles. The practical approach is to use a smoothing function of time to account for these patterns. Either local averaging techniques or various forms of smoothing or penalized splines $(36,37)$ can be used for this purpose with single profiles of observations; with multiple profiles, splines are especially useful because they naturally fit into a mixed-model framework $(18,28,30,31,42,45)$.

A single profile of log-spore data (e.g., for one location-year) can be modeled as:

$$
Y_{t}=\alpha+S\left(t_{t}\right)+e_{t}
$$

in which $S(\cdot)$ is a smooth function that characterizes the general change of $Y$ over time. We define the smoothing function in terms of radial basis functions, $B(\cdot)$ :

$$
S\left(t_{t}\right)=\sum_{l=1}^{p} u_{l} B\left(t_{t}-\kappa_{l}\right)
$$

where $\kappa_{l}$ are knots on the time axis, $B\left(t_{t}-\kappa_{l}\right)$ are the basis functions and represent the distance (on a possibly transformed scale) of each time value to each of the knots, and $u_{l}$ are coefficients $(18,19,36)$. Basically, $p$ points along the time axis, called knots $\left(\kappa_{l} ; l=1, \ldots, p\right)$, are defined, and the distance (or a function of the distance) between $t$ and each of the $\kappa_{l}$ values is calculated to construct $p$ different $B\left(t_{t}-\kappa_{l}\right)$ values. Model results are known to be generally insensitive to the number of knots (when model fitting is performed using mixed-model algorithms; see below), as long as there are no more than four or five time values (days) between each knot $(36,37)$; in fact, there can be a knot for every time but this generally increases the computational complexity of the model fitting without changing the mixedmodel-based smoothing results. We specified knots at 4-day intervals. Model results are also generally insensitive to the form of the basis function (36), although different authors appear to have favorite choices for $B\left(t_{t}-\kappa_{l}\right)$. We used the cubic radial basis function (15), $B\left(t_{t}-\kappa_{l}\right)=\left|t_{t}-\kappa_{l}\right|^{3}$. Schabenberger and Gotway (37) discuss some of the advantages of radial basis functions. As shown by Ruppert et al. (36) and Ngo and Wand (31), $S(\cdot)$ in equation 10 (with a radial basis functions on the right-hand side) can be reformulated into the classical form of the random-effects variance component (with a single variance term, $\sigma_{S}^{2}$ ) of a mixed model, allowing direct prediction of the $u_{t}$ effects with maximum likelihood (ML). For a single profile of observations, the estimation method is the equivalent of penalizing the $u_{t}$ terms in a least-squares (fixed-effects) algorithm so that there is a smooth fit to the data, capturing the general trends without overfitting or "connecting the points" (45).

The variance $\sigma_{S}^{2}$ determines the smoothness of the function. For a model with a simple (single) residual variance, $\sigma_{e}^{2}$, which is not the case here, a smoothing parameter is defined to be proportional to $\lambda=\sigma_{e}^{2} / \sigma_{S}^{2}$ (more specifically, a power of this variance ratio, dependent on the basis function being used). The smaller the $\sigma_{S}^{2}$, the larger the $\lambda$ and the smoother the fitted curve. Unlike the traditional approach of preassigning $\lambda$ (and, hence, the degree of smoothness obtained) (19), with ML estimation, the degree of smoothness is determined by the data. An arbitrary degree of smoothness can be justified for well-described systems, where the observations follow (more or less) consistent temporal trends; this is accomplished by fixing $\sigma_{S}^{2}$ in the mixed-model fit. However, for systems such as ours, the temporal trends are unknown prior to the analysis. Thus, the data inform the smoothing function fit in a natural way. With the chosen structure for $B(\cdot)$, use of equation 10 is known as radial smoothing, and the equation 
is referred to as an approximate low-rank thin-plate spline or, more simply, as a radial smoother $(15,36,37)$.

One useful summary statistic related numerically to $\lambda$ for models such as equation 10 is the degrees of freedom for the fit of the smoother $\left(d f_{S}\right)$ (30). As explained by Ruppert et al. (36), $d f_{S}$ is the equivalent number of parameters for a model fit, which can be compared (loosely) with the degrees of freedom of polynomial regression functions. For instance, a straight-line model $\left(\alpha+\gamma_{1} t+\right.$ $\left.e_{t}\right)$ has $1 \mathrm{df}$ for the fit and the quadratic model $\left(\alpha+\gamma_{1} t+\gamma_{2} t^{2}+e_{t}\right)$ has $2 \mathrm{df}$ for the fit. Unlike in other model fitting, $d f_{s}$ is not predetermined in likelihood-based model fitting but is estimated from the mixed-model-spline fit. Correspondingly, small values of $d f_{s}$ indicate very smooth changes in $Y$ with $t$, with little change, if any, in the sign of the slope; large values of $d f_{S}$ indicate more irregular changes in $Y$ with $t$, with possibly several changes in the sign of the slope, and the predictions of $Y$ coming closer to interpolating between observations.

Equation 10 was expanded to accommodate the group effect (in particular, fixed effects of location and the random effects of year within location) as well as the weather-variable effects (with a polynomial constraint on the $\beta_{m}$ parameters) and correlated residuals. The model is written as a generalization of equation 9:

$$
\begin{aligned}
Y_{i j t}= & \alpha+\sum_{k=0}^{d} \eta_{k}^{(1)} Z_{(k) i j t}^{(1)}+\sum_{k=0}^{d} \eta_{k}^{(2)} Z_{(k) i j t}^{(2)}+\sum_{k=0}^{d} \eta_{k}^{(3)} Z_{(k) i j t}^{(3)} \\
& +S_{i j}\left(t_{t}\right)+L_{i}+T(L)_{i j}+e_{i j t}
\end{aligned}
$$

Note that the $S_{i j}(\cdot)$ notation indicates that the coefficients of the radial smoother depend on the location-year ( $i j$ combination). This is analogous to there being an interaction of location-year (the grouping factor) and the smooth function of time in the model. Equation 12 was used in two forms, with and without the weather-variable predictors. This equation is known sometimes as a semiparametric (regression) mixed model, and the smoothing function is known as a nonparametric component of the model (36).

Model fitting. Basic mixed models and PDLs. We fitted versions of equation 9 to the data using ML, utilizing the GLIMMIX procedure of SAS (SAS Inc., Cary, NC), after first constructing the required $Z_{(k)}$ variables (for different polynomial degrees for the different weather predictors; see Appendix) in a data step of SAS. We also fitted equation 8 to the data using the two-stage approach described above and by Paul et al. (33). ML estimation was used instead of restricted ML so that direct comparisons of log-likelihoods could be made for models with different fixed effects (i.e., models with different weather variables) as well as different random effects (26). Standard errors are slightly biased with ML but the bias would be low with the number of observations used in this analysis (26). Initially, unlike in Paul et al. (33), all combinations of one to six predictor variables in the model were considered in separate model fits, in order to compare the relative degree of fit of the different models. A time window of $n=9$ days was used for all analyses (i.e., the current and the previous 8 days). We could have explored different values for $n$ but chose to focus on other model comparisons with a given window length. The polynomial degree (equation 2) for each predictor variable was kept the same as found in Paul et al. (33), at least for this phase of the analysis. The degrees $(d)$ were 0 for INTENSITY and AT15RH90, 1 for AVARH and AVDARH, and 2 for AVDAT and AT1530. All terms in the model were fixed effects, except for $T(L)_{i j}$ and $e_{i j t}$, which were random effects. To account for (potential) serial correlation of the residuals within each location-year (repeated-measures nature of the data), beyond that accounted for by the inclusion of the $T(L)_{i j}$ term, an order-7 Toeplitz residual covariance structure was used (26). This is a moving-average type of structure, where there is a non-zero correlation (beyond that specified through the $T(L)_{i j}$ term) to the residuals within a location-year that are up to 6 days apart.
Following the protocols for multimodel inference based on information-theoretic principles, developed by Burnham and Anderson $(7,8)$, the finite-sample version of the Akaike Information Criterion (AICC) was calculated and stored for each model fit. The AICC is a function of the log-likelihood estimated for the model fit and a penalty for the number of estimated parameters. The smaller the AICC, the better the fit. AICC is an estimate of the relative expected Kullback-Leibler (K-L) information, and is directly related to the information loss when a model is fitted to data $(7,8)$.

The minimum AICC for a collection of fitted models is given by $\mathrm{AICC}_{\text {min }}$; the model corresponding to $\mathrm{AICC}_{\text {min }}$ provides the best fit (of those models being considered) in terms of this information criterion. The difference between $\mathrm{AICC}_{\text {min }}$ and the AICC for each model is a measure of the goodness of fit. This is written as $\Delta_{r}=\mathrm{AICC}_{r}-\mathrm{AICC}_{\text {min }}$, where $r$ is an index used to refer to the individual models when $R$ different models are fitted ( $r=1$, $\ldots, R)$. The smaller the $\Delta_{r}$, the better the fit relative to the bestfitting model. With six different predictor variables, there are $R=$ 63 models. By fitting all possible models (as a foundation for later selection of the better fits), one can directly measure the evidence favoring any single model over other models under consideration. The weight of evidence in favor of one model over another is conveniently determined by rescaling the $\Delta_{r}$ values as (8):

$$
o_{r}=\frac{\exp \left(-\Delta_{r} / 2\right)}{\sum \exp \left(-\Delta_{r} / 2\right)}
$$

The denominator is simply the total of all the transformed $\Delta_{r}$ values; the sum of the model weights $\left(\Sigma o_{r}\right)$ equals 1 . The exponential function puts the goodness-of-fit measurements on the same scale as the original observations. There is a direct interpretation of the weights: $o_{r}$ is the estimated probability that the $r$ th model is the best (based on the information criterion) among the set of $R$ candidate models considered for the data being analyzed. For instance, if $o_{1}=0.4$, then this model has a $40 \%$ chance of being the best model in the set. The ratio of the weights for different models is also useful heuristically; for instance, if the first model has the highest model weight, with $o_{1}=0.4$, and the third model has $o_{3}=0.25$, then the first model is 1.6 $(0.4 / 0.25)$ times more likely than the third model of being the best in the set.

Estimates of $\alpha$ and the $\eta_{k}$ fixed-effect parameters (and their estimated standard errors [SEs]), as well as predictions of the random-effect coefficients (components of $T(L)$ and $e$ ), were obtained with each model fit. An $F$ test was used to determine whether there was an overall significant relationship between a weather variable and the response variable. For instance, if the polynomial degree is $d=2$ for predictor variable $X$ (suppressing the location-year-time subscripts and the superscript to identify a particular weather variable), there are three $\eta_{k}$ parameters: $\eta_{0}, \eta_{1}$, and $\eta_{2}$ (see equation 3). Simultaneous equality of the three $\eta_{k}$ parameters to 0 is an indication that the predictor is not related to the response. The simultaneous test of the three contrasts (in this illustration) was achieved using the contrast statement in the GLIMMIX procedure (26).

Interest is primarily in the estimated $\beta_{m}$ parameters (once again suppressing superscripts), and these were obtained from estimates of the $\eta_{k}$ parameters through the use of equation 2 . The estimated marginal effect of a weather variable $\left(\hat{\beta}^{*}\right)$ was estimated by the summation of the estimated $\beta_{m}$ parameters (for each predictor variable). These estimates and their SEs were obtained through the use of estimate statements in the GLIMMIX procedure. Student $t$ tests were used to determine if a marginal effect was significantly different from 0 .

Prediction of $Y$ were obtained with the estimated parameters and the predicted values of the random effects in the models. These predictions are estimated best unbiased linear predictors 
(EBLUPs) of the log daily spore densities (26). From these predictions, the concordance correlation coefficient (CCC) (27) was calculated to determine the agreement between observed and predicted log daily spore densities (the closeness of the EBLUPs to the observations). CCC is one of several possible measures of goodness of fit for mixed models (27).

Mixed model expansions. The polynomial degree for each weather variable was chosen a priori based on the individual PDL regression results (33). However, when the correlations among the predictors, the correlations among residuals over time within location-years, and random effects are taken into account (through the full mixed-model formulation; equation 9), other polynomial degrees may be warranted. As discussed by Batten and Thornton (4), using an overly low value of $d$ in the polynomial of equation 2 will result in biased parameter estimates. Thus, for the best-fitting models based on the a priori polynomial degrees (see Results), we considered versions of equation 9 with one higher value of $d$ than used by Paul et al. (42), and fitted these models with ML as described above. Goodness of model fit was based on the AICC (8) statistic and the CCC (27). Student $t$ tests were used to see if the parameter for the added higher-order $\eta_{k}$ term was significantly different from 0 .

Radial smoothing. Equation 12 without weather variables was fitted to the data using ML, as described by Ruppert et al. (36) and Ngo and Wand (31), utilizing the new smoothing-function options in the GLIMMIX procedure of SAS. Then, versions of equation 12 were fitted which corresponded to the best-fitting mixed models without the smoothing function (equation 9; see Results). Moreover, versions of equation 12 with one higher polynomial degree $(d)$ for the weather variables were also fitted.

The AICC was determined for each model fit and CCC was calculated for the agreement between observed and predicted log daily spore densities. Differences of AICC values for different models were also calculated for the set of models under consideration.

\section{RESULTS}

PDL mixed models. Versions of the PDL mixed model in equation 9, consisting of one to six predictor weather variables, were successfully fitted to the example data (log daily spore densities of G. zeae) using ML. The model comprised of temporal-lagged functions of AVARH, AVDAT, and INTENSITY provided the best overall fit, of the 63 models fitted, in terms of the AICC likelihood-based statistic (Table 1). When based on composite weather variables (equation 8), the model with these three predictors was labeled M1 by Paul et al. (33). We retain the same general model-number labeling for the use of equation 9 (with no constraint on the $\beta_{m}$ [or weight] values other than they fall on the curve specified by the choice of $d$ ). However, to be more specific, we add a c subscript to the model label (e.g., M1 $1_{c}$ ) when referring to a model based on composite predictor variables (equation 8), and an $\mathrm{f}$ subscript (e.g., $\mathrm{M} 1_{\mathrm{f}}$ ) when referring to the full mixed model (equation 9). The second-best-fitting model (characterized by AICC) comprised temporal-lagged functions of AVARH, AT1530, and INTENSITY (Table 1). This is referred to as model $\mathrm{M} 3_{\mathrm{f}}$. The PDL full mixed-model versions of the other models in Table 4 of Paul et al. (33) provided markedly poorer fits to the data, as quantified by the AICC (Table 1). However, all weather variables in all five of these models were significant $(P<$ 0.05 ) based on $F$ tests.

The four models that gave the lowest AICC values after models $\mathrm{M} 1_{\mathrm{f}}$ and $\mathrm{M} 3_{\mathrm{f}}$ are also listed in Table 1 . These all involved rainfall (as INTENSITY) and some form of relative humidity (as AVARH in most cases, or as AVDARH) and temperature (as AVDAT in most cases, but also AT1530 and AT15RH90 [which also involved relative humidity]). Three of these comprised four weather predictor variables; however, at least one of the predictors was not significant $(P>0.2)$ in each four-predictor model. The lack of significance was due to the correlation among some of the predictor variables. Moreover, the AICC values for the four-predictor models were higher than for the best three-predictor models, indicating degradation in the fit to the data relative to the number of parameters estimated.

In comparison, using models with composite weather variables (equation 8) and ML estimation, an AICC of 1,657.7 was found for $\mathrm{M} 1_{\mathrm{c}}$ and 1,656.1 for $\mathrm{M} 3_{\mathrm{c}}$. These statistics are similar but slightly below the values found here for the full linear mixed models (equation 9) (Table 1), although the direct comparison of these parameter-penalized goodness-of-fit statistics between equations 8 and 9 is problematic. In equation 9 (with either $M 1_{\mathrm{f}}$ or $\mathrm{M}_{\mathrm{f}}$ ), there are six parameters for the weather predictors (one for INTENSITY [because $d=0$ ], two for AVARH [ $d=1$ ], and three for the temperature variables $[d=2])$; in contrast, there are only three explicit parameters for equation 8 (one for each composite predictor). However, the composite weather variables were calculated using weights that were obtained from separate PDL regressions, with from one to three $Z_{(k)}$ variables for each weather variable; thus, there are "hidden" parameters in equation 8 that are not accounted for in the routine calculation of AICC. By using a comparable parameter-penalty adjustment to the loglikelihood (i.e., by accounting for the hidden parameters), we found that AICC was 1,665.7 for model $\mathrm{M} 1_{c}$ with composite weather variables and 1,663.0 for model $\mathrm{M} 3_{\mathrm{c}}$ with composite weather variables, both slightly higher than that found for $M 1_{f}$ and $\mathrm{M} 3_{\mathrm{f}}$.

TABLE 1. Statistics for linear mixed models (equation 9) fitted to the log of daily spore density of Gibberella zeae on wheat spikes, including the corrected Akaike Information Criterion (AICC), model weight $\left(o_{r}\right)$ based on AICC (see equation 13), ratio of $o_{r}$ to the smallest $o_{r}$ (in the set of models being considered), and concordance correlation coefficient (CCC)

\begin{tabular}{|c|c|c|c|c|}
\hline Model $^{\mathrm{a}}$ & AICC & Model weight & Weight ratio & $\mathrm{CCC}$ \\
\hline $\mathrm{M1}_{\mathrm{f}}:$ AVARH, AVDAT, INTENSITY & $1,660.1$ & 0.230 & & 0.790 \\
\hline M2 $2_{\mathrm{f}}$ : AVDAT, AVDARH, INTENSITY & $1,672.9$ & $<0.001$ & 621.6 & 0.784 \\
\hline M3 $3_{\mathrm{f}}$ : AVARH, AT1530, INTENSITY & $1,660.7$ & 0.171 & 1.4 & 0.783 \\
\hline M5 & $1,677.6$ & $<0.001$ & 575.0 & 0.766 \\
\hline AVARH, AVDAT, INTENSITY, AT15RH90 & $1,661.5$ & 0.114 & 2.0 & 0.790 \\
\hline AVARH, AVDAT, INTENSITY, AT1530 & $1,662.6$ & 0.065 & 3.5 & 0.791 \\
\hline AVARH, INTENSITY, AT1530, AT15RH90 & $1,662.7$ & 0.062 & 3.7 & 0.782 \\
\hline
\end{tabular}

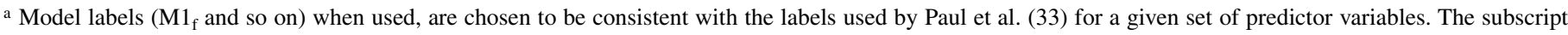
"f" indicates that these models were based on the full linear mixed model (without composite variables). A model label is not used if there is not a corresponding model in Paul et al. (33) Variables listed are the predictor variables used in the specified model. In all cases, the effect of each predictor was distributed over 9 days (the current day and time lags of 1 to 8 days). Polynomial order was $d=0$ for daily average rainfall intensity (INTENSITY) and number of hours per day with air temperature $\geq 15^{\circ} \mathrm{C}$ and relative humidity $\geq 90 \%$ (AT15RH90), $d=1$ for average daily relative humidity of the air (AVARH) and average daytime $(0600$ to $1800 \mathrm{~h})$ relative humidity of the air (AVDARH), and $d=2$ for average daytime air temperature $\left({ }^{\circ} \mathrm{C}\right)(\mathrm{AVDAT})$ and number of hours per day with air temperature of 15 to $30^{\circ} \mathrm{C}$ (AT1530). See text in Materials and Methods for description of the predictors. 
As measured by CCC (27), all of the models in Table 1 had similar accuracy in predicting log of daily spore densities. Models $\mathrm{M} 1_{\mathrm{f}}$ and two of the four-predictor models had slightly larger CCC values than the next best models. We did not further consider the four-predictor-variable models because of nonsignificant predictors. The CCC values were not of value in discriminating among models (of those presented here) because they were so similar among the different models. However, the model weights $\left(o_{f}\right.$; equation 13) and the weight ratio (weight of each model relative to the weight for the best fitting model [i.e., $\mathrm{M} 1_{\mathrm{f}}$ ]) allowed clear separation of these models. The probability that model $\mathrm{M}_{\mathrm{f}}$ was the best model (of those being considered) was estimated to be 0.23 ; for model $\mathrm{M}_{\mathrm{f}}$, the estimated probability that it was the best model was 0.17 (Table 1). The estimated probabilities were $<0.11$ for the other models. Of the models not presented in the table, none had a weight $>0.04$ and $85 \%$ had weights $<0.01$. Model $M 1_{\mathrm{f}}$

TABLE 2. Estimated $\eta_{k}$ parameters for the constructed $Z_{(\mathrm{k})}$ variables for models $\mathrm{M}_{\mathrm{f}}, \mathrm{M}_{\mathrm{f}}, \mathrm{M}_{\mathrm{f}}-\mathrm{S}$, and $\mathrm{M} 3_{\mathrm{f}}-\mathrm{S}^{\mathrm{a}}$

\begin{tabular}{lcccc}
\hline & \multicolumn{4}{c}{ Model } \\
\cline { 2 - 5 } Parameter $^{\mathrm{b}}$ & $\mathrm{M} 1_{\mathrm{f}}$ & $\mathrm{M} 3_{\mathrm{f}}$ & $\mathrm{M} 1_{\mathrm{f}}-\mathrm{S}$ & $\mathrm{M} 3_{\mathrm{f}}-\mathrm{S}$ \\
\hline$\hat{\eta}_{1}^{(1)}$ & 0.0104 & 0.0106 & 0.0103 & 0.0107 \\
$\hat{\eta}_{2}^{(1)}$ & -0.00076 & -0.00086 & -0.00068 & -0.00083 \\
$\hat{\eta}_{1}^{(2)}$ & -0.0115 & -0.0093 & -0.0182 & -0.0132 \\
$\hat{\eta}_{2}^{(2)}$ & 0.0144 & 0.0095 & 0.0154 & 0.0090 \\
$\hat{\eta}_{3}^{(2)}$ & -0.00171 & -0.00113 & -0.00180 & -0.00105 \\
$\hat{\eta}_{1}^{(3)}$ & 0.0330 & 0.0322 & 0.0258 & 0.0273 \\
\hline
\end{tabular}

${ }^{a}$ Models $\mathrm{M}_{\mathrm{f}}, \mathrm{M}_{\mathrm{f}}$ are defined using equation 9 and models $\mathrm{M} 1_{\mathrm{f}}-\mathrm{S}$ and $\mathrm{M}_{\mathrm{f}}-\mathrm{S}$ are defined using equation 12 .

${ }^{b}$ For the parameter estimates, the "(1)" superscript refers to the predictor average daily relative humidity of the air and the "(3)" superscript refers to the daily average rainfall intensity predictor. The "(2)" superscript refers to the average daytime air temperature $\left({ }^{\circ} \mathrm{C}\right)$ predictor for models $\mathrm{M}_{\mathrm{f}}$ and $\mathrm{M} 1_{\mathrm{f}} \mathrm{S}$, and to the number of hours per day with air temperature of 15 to $30^{\circ} \mathrm{C}$ predictor for models $\mathrm{M}_{\mathrm{f}}$ and $\mathrm{M}_{\mathrm{f}}-\mathrm{S}$. The number of parameters for a predictor variable depends on the polynomial order $(d)$ being used. There are $d+1$ parameters for a given weather variable. was 1.4 times more likely to be the best model than was $\mathrm{M}_{\mathrm{f}}$. Model $\mathrm{M} 1_{\mathrm{f}}$ was also several-hundred-fold more likely to be the best model than the other mixed-model formulations explicitly considered as examples by Paul et al. (33) (models $\mathbf{M} 2_{\mathrm{f}}, \mathbf{M} 4_{\mathrm{f}}$, and $\mathrm{M} 5_{\mathrm{f}}$ ). Furthermore, Model $\mathrm{M} 1_{\mathrm{f}}$ was 4.3-times more likely to be the best model than the other three-predictor-variable models with a relatively low AICC in Table 1 (AVDARH, AVDAT, and INTENSITY).

Estimated parameters and weights of the fitted models. Based on the combined results summarized in Table 1, the remainder of this article deals primarily with models $M 1_{f}$ and $M 3_{f}$ and their expansions and generalizations. The estimated $\eta_{k}$ parameters of the constructed variables of equation 9 for these two models are given in Table 2. However, interest and interpretation primarily focuses on the estimated $\beta_{m}$ parameters derived from the estimated $\eta_{k}$ values (i.e., $\hat{\beta}_{m}^{(1)}, \hat{\beta}_{m}^{(2)}, \hat{\beta}_{m}^{(3)}$; see equation 2), the estimated location effects, and overall intercept $\left(\hat{L}_{i}, \hat{\alpha}\right)$. These parameter estimates, as well as the estimated marginal-effect parameters (e.g., $\hat{\beta}^{(1)^{*}}$ ), are given in Table 3. For instance, the estimated marginal effect of the first weather predictor (AVARH) is calculated as:

$$
\hat{\beta}^{(1)^{*}}=\sum_{m}^{n} \hat{\beta}_{m}^{(1)}=65.8 \times 10^{-3}
$$

with $m$ ranging from 0 to $n(=8)$. Note that, in Table 3 , the parameter estimates are multiplied by $10^{3}$ for ease of presentation. The estimated daily temporal lag parameters, and also the marginal-effect parameters, for AVARH and INTENSITY were similar between models $\mathrm{M}_{\mathrm{f}}$ and $\mathrm{M}_{\mathrm{f}}$ (both of which contained these weather variables). Furthermore, the estimated marginal effects for AVARH and INTENSITY found from fitting the full mixed model here (equation 9; Table 3) were similar to the estimates found in fitting the mixed model with composite weather variables $\left(\mathrm{M} 1_{\mathrm{c}}\right.$ and $\mathrm{M} 3_{\mathrm{c}}$; see equation 8$)$. In particular, using composite variables and the two-stage process, $\hat{\beta}^{(1)^{*}}=62 \times 10^{-3}$ for AVARH and $\hat{\beta}^{(3)^{*}}=306 \times 10^{-3}$ for INTENSITY with model $M 1_{c}$ and $\hat{\beta}^{(1)^{*}}=64 \times 10^{-3}$ for AVARH and $\hat{\beta}^{(3)^{*}}=288 \times 10^{-3}$ for INTENSITY for model M3 .

TABLE 3. Estimated (fixed-effects) parameters for two linear mixed models $\left(\mathrm{M}_{\mathrm{f}}\right.$ and $\mathrm{M}_{\mathrm{f}}$; see equation 9$)$ fitted to the log of daily spore density $(Y)$ of Gibberella $z e a e$ on wheat spikes, where $Y$ on a given day is affected by the predictor (weather) variables on the current $(t)$ and 8 previous days $(t-1 \text { to } t-8)^{\mathrm{a}}$

\begin{tabular}{|c|c|c|c|c|c|c|c|c|}
\hline \multirow[b]{2}{*}{ Time lag } & \multicolumn{4}{|c|}{ Model $\mathrm{M} 1_{\mathrm{f}}^{\mathrm{b}}$} & \multicolumn{4}{|c|}{ Model M3 ${ }_{\mathrm{f}}^{\mathrm{b}}$} \\
\hline & $\begin{array}{l}\text { AVARH } \\
\left(\times 10^{3}\right)\end{array}$ & $\begin{array}{l}\text { AVDAT } \\
\left(\times 10^{3}\right)\end{array}$ & $\begin{array}{l}\text { INTENSITY } \\
\quad\left(\times 10^{3}\right)\end{array}$ & $\begin{array}{l}\text { Additional } \\
\text { constants }^{\mathrm{c}}\end{array}$ & $\begin{array}{l}\text { AVARH } \\
\left(\times 10^{3}\right)\end{array}$ & $\begin{array}{l}\text { AT1530 } \\
\left(\times 10^{3}\right)\end{array}$ & $\begin{array}{c}\text { INTENSITY } \\
\left(\times 10^{3}\right)\end{array}$ & $\begin{array}{l}\text { Additional } \\
\text { constants }^{c}\end{array}$ \\
\hline$t$ & $10.4(2.9)$ & $-11.5(9.3)$ & $33.0(10.3)$ & $\ldots$ & $10.6(2.8)$ & $-9.3(5.5)$ & $32.2(10.5)$ & $\ldots$ \\
\hline$t-1$ & $9.6(2.4)$ & $1.2(6.0)$ & $33.0(10.3)$ & $\ldots$ & $9.8(2.4)$ & $-0.9(3.9)$ & $32.2(10.5)$ & $\ldots$ \\
\hline$t-2$ & $8.8(2.1)$ & $10.4(4.8)$ & $33.0(10.3)$ & $\ldots$ & $8.9(2.1)$ & $5.2(3.4)$ & $32.2(10.5)$ & $\ldots$ \\
\hline$t-3$ & $8.1(1.8)$ & $16.2(5.0)$ & $33.0(10.3)$ & $\ldots$ & $8.0(1.9)$ & $9.0(3.5)$ & $32.2(10.5)$ & $\ldots$ \\
\hline$t-4$ & $7.3(1.7)$ & $18.6(5.3)$ & $33.0(10.3)$ & $\ldots$ & $7.2(1.8)$ & $10.6(3.6)$ & $32.2(10.5)$ & $\ldots$ \\
\hline$t-5$ & $6.6(1.8)$ & $17.5(5.2)$ & $33.0(10.3)$ & $\ldots$ & $6.3(1.8)$ & $9.9(3.5)$ & $32.2(10.5)$ & $\ldots$ \\
\hline$t-6$ & $5.8(1.9)$ & $13.0(5.0)$ & $33.0(10.3)$ & $\ldots$ & $5.5(2.0)$ & $6.9(3.2)$ & $32.2(10.5)$ & $\ldots$ \\
\hline$t-7$ & $5.0(2.2)$ & $5.1(5.8)$ & $33.0(10.3)$ & $\ldots$ & $4.6(2.2)$ & $1.7(3.6)$ & $32.2(10.5)$ & $\ldots$ \\
\hline$t-8$ & $4.3(2.6)$ & $-6.2(8.6)$ & $33.0(10.3)$ & $\ldots$ & $3.7(2.6)$ & $-5.7(5.0)$ & $32.2(10.5)$ & $\ldots$ \\
\hline Marginal $^{\mathrm{d}}$ & $65.8(15.7)$ & $64.4(32.5)$ & $297.1(93.0)$ & $\ldots$ & $64.6(15.8)$ & $27.3(20.3)$ & $289.8(94.9)$ & $\ldots$ \\
\hline Intercept & $\ldots$ & $\ldots$ & $\ldots$ & $-1.6(1.4)$ & $\ldots$ & $\ldots$ & $\ldots$ & $-0.6(1.3)$ \\
\hline \multicolumn{9}{|l|}{ Location } \\
\hline Indiana & $\ldots$ & $\ldots$ & $\ldots$ & $-2.3(0.7)$ & $\ldots$ & $\ldots$ & $\ldots$ & $-2.4(0.6)$ \\
\hline Manitoba & $\ldots$ & $\ldots$ & $\ldots$ & $-1.1(1.1)$ & $\ldots$ & $\ldots$ & $\ldots$ & $-1.1(1.1)$ \\
\hline North Dakota & $\ldots$ & $\ldots$ & $\ldots$ & $-2.3(0.6)$ & $\ldots$ & $\ldots$ & $\ldots$ & $-2.4(0.6)$ \\
\hline Ohio & $\ldots$ & $\ldots$ & $\ldots$ & $-2.5(0.6)$ & $\ldots$ & $\ldots$ & $\ldots$ & $-2.6(0.6)$ \\
\hline Pennsylvania & $\ldots$ & $\ldots$ & $\ldots$ & $-2.1(0.7)$ & $\ldots$ & $\ldots$ & $\ldots$ & $-2.2(0.7)$ \\
\hline South Dakota & $\ldots$ & $\ldots$ & $\ldots$ & 0 & $\ldots$ & $\ldots$ & $\ldots$ & 0 \\
\hline
\end{tabular}

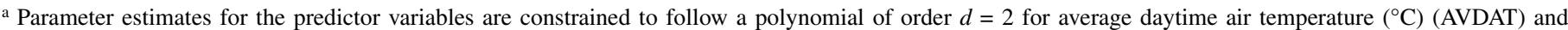
number of hours per day with air temperature of 15 to $30^{\circ} \mathrm{C}$ (AT1530), $d=1$ for average daily relative humidity of the air (AVARH), or $d=0$ for daily average rainfall intensity (INTENSITY). See text for description of the variables.

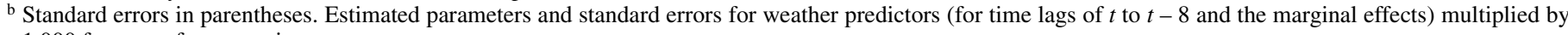
1,000 for ease of presentation.

c Estimates of $\alpha$ (intercept) and the $L_{i}$ location effects in equation 9. All effects are on a log scale.

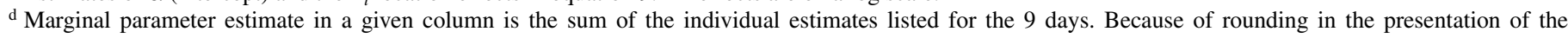
individual estimates, the sum of the shown time-lag coefficients may be slightly different from the presented estimated marginal effect (at the last decimal place). 
The marginal effect estimated here with equation 9 for AVDAT $\left(\hat{\beta}^{(2)^{*}}=64.4 \times 10^{-3}\right.$; see model $\mathrm{M} 1_{\mathrm{f}}$ in Table 3 ) was substantially higher than that estimated using the composite weather variable $\left(\hat{\beta}^{(2)^{*}}=17 \times 10^{-3}\right.$; model M1 $)$. The estimated marginal effect for AT1530 in model $\mathrm{M} 3_{\mathrm{f}}\left(\hat{\beta}^{(2)^{*}}=27.3 \times 10^{-3}\right.$; Table 2$)$ was similar to that estimated using the composite weather variable in $\mathrm{M} 3_{\mathrm{c}}$ $\left(\hat{\beta}^{(2)^{*}}=30 \times 10^{-3}\right)$. Note that the weights with composite weather variables are determined before the mixed model is fitted (and that only the marginal-effect parameter is estimated for the composite variable) but the weights for the full mixed model (e.g., $\hat{w}_{m}^{(2)}=\hat{\beta}_{m}^{(2)} / \hat{\beta}^{(2)^{*}}$ ) are estimated as a part of the actual model fit (through the estimated $\beta_{m}$ values). The estimated weights for all the variables in models 1 and 3, except for INTENSITY, are shown in Figure 1A and 1B, based on the fit of the full PDL mixed model $\left(\mathrm{M} 1_{\mathrm{f}}\right.$ and $\mathrm{M} 3_{\mathrm{f}}$; equation 9), together with the weights used in mixed models with composite weather variables $\left(\mathrm{M} 1_{\mathrm{c}}\right.$ and $\mathrm{M} 3_{\mathrm{c}}$; equation 8$)$. Weights are equal (=1/9) at all lags for INTENSITY because of the 0 polynomial degree and are not shown on the graph. The estimated weights were very similar with the two modeling approaches for AVARH for both models
$\mathrm{M} 1_{\mathrm{f}} / \mathrm{M} 3_{\mathrm{c}}$ (Fig. 1A) and $\mathrm{M} 3_{\mathrm{f}} / \mathrm{M} 3_{\mathrm{c}}$ (Fig. 1B). There was a very slight tendency for the weights with the composite variable to be higher at short lags and lower at long lags. The greatest weight was given to the current day and the weights declined linearly (as required, based on $d=1$ ) with lag. All weights were positive. Thus, the modeling approach (one- or two-stage) did not have a discernable effect on the results for AVARH.

The estimated weights for AVDAT followed a quadratic trend with temporal lag, as required (by the choice of $d$ ), and the weights were either positive or negative, with a negative relation between $Y$ and AVDAT on the current day and a positive relation at moderate lags (especially for lags of 3 to 6) (Fig. 1A). However, the change in weights was much greater for the model with a composite predictor $\left(\mathrm{M} 3_{\mathrm{c}}\right)$ than for the full PDL mixed model $\left(\mathrm{M}_{\mathrm{f}}\right)$. At a lag of 0 (the current day), there was a larger negative weight given to AVDAT when composite weather variables were used compared with when the full PDL mixed model was fitted; at moderate temporal lags, there was a larger positive weight for the model with the composite weather variables compared with when the full PDL mixed model was fitted (Fig.
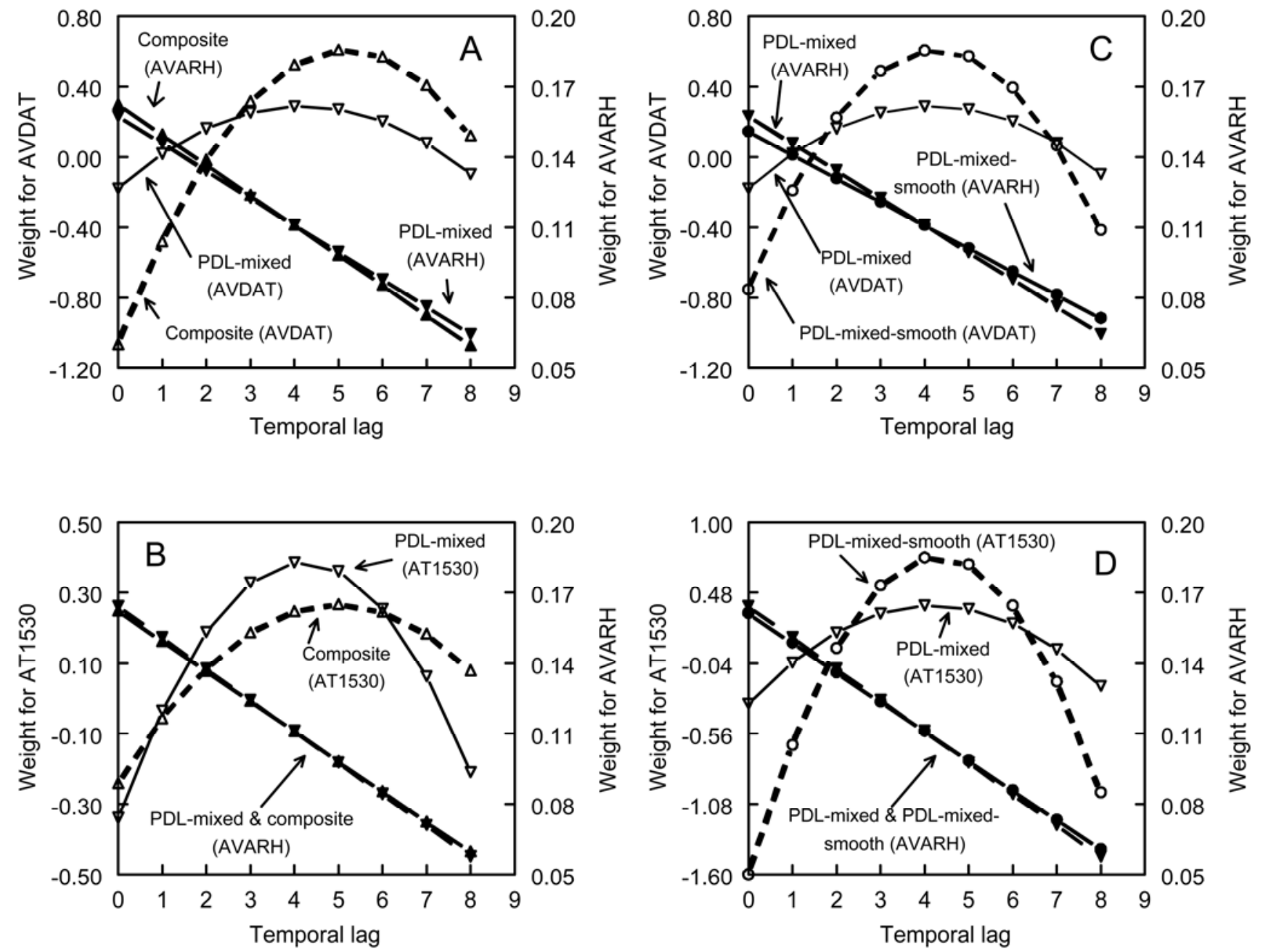

Fig. 1. Estimated time-lag weights of predictor weather variables (e.g., $\hat{w}_{m}^{(2)}=\hat{\beta}_{m}^{(2)} / \hat{\beta}^{(2)^{*}}$ ) for the second predictor variable, where $\hat{\beta}^{(2)^{*}}=\sum \hat{\beta}_{m}^{(2)}$ ) for the fit of a full linear mixed model (with either a radial smooth function [equation 12] or no smooth function [equation 9]) to the log of daily spore density ( $Y$ ) of Gibberella zeae on wheat spikes, or prespecified weights for the construction of composite weather variables (equation 5) when equation 8 was fitted to the data. A, Weights for average daily relative humidity of the air (AVARH) (right-hand axis) and average daytime air temperature $\left({ }^{\circ} \mathrm{C}\right)$ (AVDAT) (left-hand axis) for model $\mathrm{M} 1_{\mathrm{f}}$, based on the fit of the full linear mixed model (equation 9, polynomial distributed lag [PDL]-mixed), or model $\mathrm{M}_{c}$, based on the prespecified weights used to determine composite weather variables (equation 8, composite). B, Weights for AVARH (right-hand axis) and number of hours per day with air temperature of 15 to $30^{\circ} \mathrm{C}$ (AT1530) (left-hand axis) for model $\mathrm{M}_{\mathrm{f}}$, based on the fit of the full linear mixed model (equation 9, PDL-mixed), or model M3 , based on the pre-specified weights used to determine composite weather variables (equation 8, composite). C, Weights for AVARH (right-hand side) and AVDAT (left-hand side) for model $\mathrm{M1}_{\mathrm{f}}$ (equation 9, PDL-mixed), or model $\mathrm{M1}_{\mathrm{f}}-\mathrm{S}$ (equation 12, PDL-mixed-smooth). D, Weights for AVARH (right-hand axis) and AT1530 (left-hand axis) for model $\mathrm{M}_{\mathrm{f}}$, based on either the fit of the full linear mixed model (equation 9, PDL-mixed), or model $\mathrm{M} 3_{\mathrm{f}}-\mathrm{S}$ (equation 12, PDL-mixed-smooth). Scale of the vertical axis was allowed to vary among the four graphs to better show the range of estimated weights for a particular predictor variable and model. 
1A). Because of the large negative weight at lag 0 (and the more moderate negative weight at lag 1) for the model with composite weather variables, the estimated marginal effect for AVDAT was lower when composite weather variables were used $\left(\mathrm{M} 3_{\mathrm{c}}\right.$; equation 8) than when the magnitude of the $\beta_{m}$ parameters were allowed to vary freely in the estimation process $\left(\mathrm{M}_{\mathrm{f}}\right.$; equation 9$)$, subject only to the constraint that the parameters fall on a quadratic curve.

The estimated weights for AT1530 varied less between the two modeling approaches (model $\mathrm{M} 3_{\mathrm{f}}$ or $\mathrm{M} 3_{\mathrm{c}}$ ) compared with the differences in weights found for AVDAT (Fig. 1B). For AT1530, the estimated weights were higher at moderate lags and lower (more negative) at longer lags, when the full PDL model was fitted $\left(\mathrm{M} 3_{\mathrm{f}}\right)$, compared with when composite predictors were used $\left(\mathrm{M} 3_{\mathrm{c}}\right)$. The overall outcome was a slightly lower marginal effect estimate for AT1530 with the full PDL mixed model.

Standardized parameter estimates. Because the predictor variables were not on the same scale, one cannot use the estimated marginal effect parameters (or the individual time-lag parameters) to compare the relative importance of the different variables within a model in terms of predicting $Y$. One possible standardization that would allow approximate comparisons is to multiply each parameter estimate by the standard deviation of the corresponding predictor variable (across all data points). The standardized marginal effects for AVARH, AVDAT, and INTENSITY for model $\mathrm{M}_{\mathrm{f}}$ were estimated in this manner as $0.71,0.28$, and 0.78 , respectively. The standardized marginal effects for AVARH, AT1530, and INTENSITY for model $\mathrm{M} 3_{\mathrm{f}}$ were estimated as $0.70,0.18$, and 0.76 , respectively. This suggests that the temperature variables were less important in predicting $Y$ than the other weather variables, and AVARH and INTENSITY were roughly of equal importance.

PDL mixed-model generalizations. When one higher polynomial order was used for the predictors in model $\mathrm{M} 1_{\mathrm{f}}(d=2$ for AVARH, $d=3$ for AVDAT, and $d=1$ for INTENSITY), an AICC of 1,663.8 was found when the PDL mixed model was fitted to the data. This AICC is 3.7 points higher than when $\mathrm{M} 1_{\mathrm{f}}$ was fitted at the nominal values of $d$ (Table 1). Likewise, AICC was 1,661.9 when model $\mathrm{M} 3_{\mathrm{f}}$ was fitted to the data using one higher polynomial order ( $d=2$ for AVARH, $d=3$ for AT1530, and $d=1$ for INTENSITY). This AICC is 1.2 higher than when $\mathrm{M}_{\mathrm{f}}$ was fitted with nominal $d$ values. Moreover, $\mathrm{CCC}$ was unchanged (to two decimal places) compared with the nominal situation. Thus, there was no evidence with this data set to change the nominal values of the polynomial order (see equation 3 ) in the mixed models when accounting for random effects of years within locations, correlations of the predictor variables, and serial correlation over time.

Radial smoothing. Equation 12, with or without the weather predictor variables, was successfully fitted to the data using ML (Table 4). Without the weather predictors, the fitted model (labeled model S) shows the overall trend (a type of macrotrend) of the response variable with time during each location-year (Fig. 2). In many location-years, predicted values of $Y$ revealed either a general (slow) upward trend or no apparent general increase or decrease over time. There were a few cases with a more rapid increase over time (e.g., South Dakota 2001) or a general decline over time (e.g., North Dakota 2000). Furthermore, there was little visible evidence of clear-cut changes from positive to negative trends (or vice versa) within the same location-year, with one of the Indiana data sets (Indiana 2001) being an exception.

The fit of the radial smoother resulted in an AICC of 1,686.6 and a CCC of 0.852 (Table 4). The estimated degrees of freedom for the $S_{i j}\left(t_{t}\right)$ portion of the fitted model, $d f_{\mathrm{S}}$, was $\approx 24$. Given that there were 27 location-years, there was $\approx 1$ degree of freedom (i.e., $23.7 / 27=0.9$ ) for each location-year (because the subject for the smoother is the location-year). This reflects the smoothness of the predictions as shown in Figure 2. Note that a mean $d f_{S}$ does not indicate that each location-year had the same smoothness in the predictions; loosely speaking, more of the $\approx 24$ degrees of freedom would be "allotted" to some location-years than to others but, overall, there were smooth temporal trends.

When equation 12 with the weather predictor variables of model $\mathrm{M} 1_{\mathrm{f}}$ (labeled model $\mathrm{M} 1_{\mathrm{f}}-\mathrm{S}$ ) was fitted to the data, an AICC of 1,649.3 was obtained (Table 4 ). This was $\approx 11$ points lower than for model $\mathrm{M} 1_{\mathrm{f}}$ (Table 1$)$. CCC for model $\mathrm{M} 1_{\mathrm{f}}-\mathrm{S}$ was 0.08 points higher than for model $\mathrm{M}_{\mathrm{f}}$ (compare Tables 1 and 4). The $d f_{S}$ value was slightly higher than for model $S(\approx 26)$, again indicating

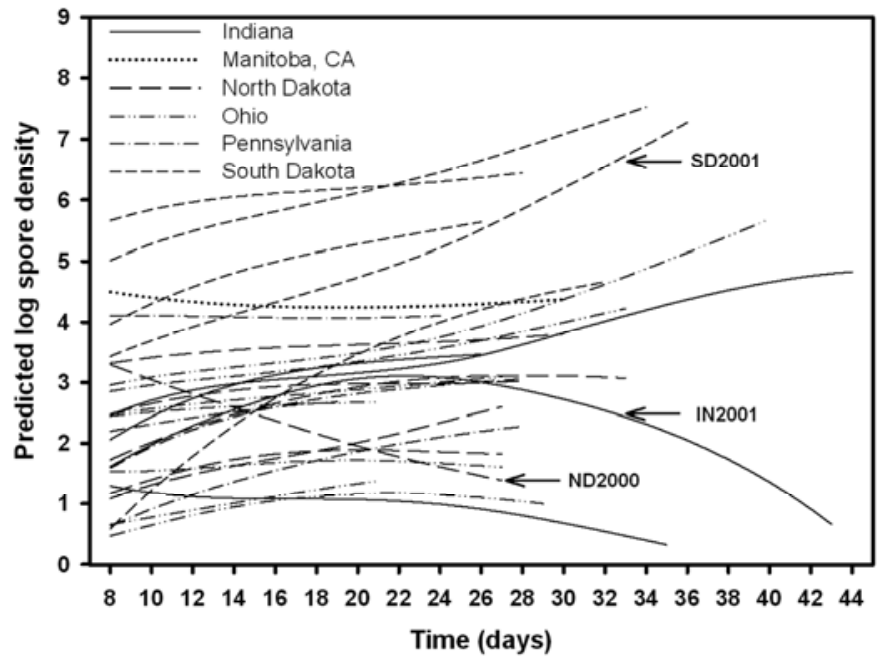

Fig. 2. Predictions of the log of daily spore density $(\hat{Y})$ of Gibberella zeae on wheat spikes for each of 27 location-years, based on the fit of the radialsmoother mixed model (equation 12) without any weather predictor variables. Degrees of freedom for the radial smoother component of the mixed model $\left(d f_{S}\right)$ and estimated variance component of the smoother are given in Table 4. SD2001, IN2001, and ND2000 refer to the predictions for South Dakota in 2001, Indiana in 2001, and North Dakota in 2000, respectively.

TABLE 4. Statistics for linear mixed models with a radial smoother (equation 12) fitted to the log of daily spore density of Gibberella zeae on wheat spikes, including the corrected Akaike Information Criterion (AICC), concordance correlation coefficient (CCC), model degrees of freedom for the radial smoother $\left(d f_{S}\right.$ ), and the estimated variance of the smoother $\left(\hat{\sigma}_{S}^{2}\right)$

\begin{tabular}{|c|c|c|c|c|}
\hline Model $^{\mathrm{a}}$ & AICC & $\mathrm{CCC}$ & $d f_{S}$ & $\hat{\sigma}_{S}^{2}\left(\times 10^{4}\right)$ \\
\hline S: Radial smoother & $1,686.6$ & 0.852 & 23.7 & 96.1 \\
\hline $\mathrm{M}_{\mathrm{f}}-\mathrm{S}: \mathrm{M} 3_{\mathrm{f}}$ plus radial smoother & $1,649.7$ & 0.867 & 25.8 & 59.4 \\
\hline $\mathrm{M}_{\mathrm{f}}-\mathrm{S}+1\left(\mathrm{M} 3_{\mathrm{f}}-\mathrm{S}\right.$, with higher polynomial degree $)$ & $1,650.5$ & 0.868 & 26.1 & 61.3 \\
\hline
\end{tabular}

a $\mathrm{S}$ : equation 12 with no weather variables. $\mathrm{M} 1_{\mathrm{f}}-\mathrm{S}$ : equation 12 with same weather predictors as in model $\mathrm{M} 1_{\mathrm{f}}$, with polynomial-order constraints of $d=2$ for average daytime air temperature $\left({ }^{\circ} \mathrm{C}\right), d=1$ for average daily relative humidity of the air $($ AVARH), and $d=0$ for daily average rainfall intensity $(\mathrm{mm} / \mathrm{h})$ (INTENSITY). M3 $3_{\mathrm{f}}-\mathrm{S}$ : equation 12 with the same weather predictors as in model $\mathrm{M}_{\mathrm{f}}$, with polynomial-order constraints of $d=2$ for number of hours per day with air temperature of 15 to $30^{\circ} \mathrm{C}, d=1$ for AVARH, and $d=0$ for INTENSITY. $1_{\mathrm{f}}-\mathrm{S}+1$ : same as model $\mathrm{M} 1_{\mathrm{f}}-\mathrm{S}$, but with one higher order $(d+1)$ to the polynomial constraints for each of the predictors. $\mathrm{M}_{\mathrm{f}}-\mathrm{S}+1$ : same as model $\mathrm{M} 3_{\mathrm{f}}-\mathrm{S}$, but with one higher order to the polynomial constraints for each of the predictors. 
$\approx 1$ degree of freedom per location-year (on average). The AICC for the fit of equation 12 with the weather predictors of model $\mathrm{M} 3_{\mathrm{f}}\left(\mathrm{M} 3_{\mathrm{f}}-\mathrm{S}\right)$ was $\approx 11$ points lower than for model $\mathrm{M} 3_{\mathrm{f}}$ (Tables 1 and 4); likewise, the concordance correlation coefficient for model $\mathrm{M} 3_{\mathrm{f}}-\mathrm{S}$ was $\approx 0.08$ points higher than for $\mathrm{M} 3_{\mathrm{f}}$. The $d f_{S}$ value was approximately the same as for $\mathrm{M} 1_{\mathrm{f}}-\mathrm{S}$.

Adding 1 degree to the polynomial functions for the time lags did not improve the fit in any manner (Table 4, models $\mathrm{M}_{\mathrm{f}}-\mathrm{S}+1$ and $\left.\mathrm{M} 3{ }_{\mathrm{f}}-\mathrm{S}+1\right)$. In fact, the AICC increased by using a higher degree; thus, these generalizations were not considered further.

In terms of goodness of fit (AICC and CCC) and smoothness of the radial function $\left(d f_{S}\right)$, there was little difference between models $\mathrm{M} 1_{\mathrm{f}}-\mathrm{S}$ and $\mathrm{M} 3_{\mathrm{f}}-\mathrm{S}$. The estimated $\eta_{k}$ parameters are given in Table 2, and the more informative parameter estimates $\left(\hat{\beta}_{m}^{(1)}, \hat{\beta}_{m}^{(2)}, \hat{\beta}_{m}^{(3)}, \hat{L}_{i}, \hat{\alpha}\right)$ for these two models are given in Table 5, together with the marginal-effects parameters (e.g., $\hat{\beta}^{(2)^{*}}$ ). From the $\beta_{m}$ parameter estimates, the temporal-lag weights were estimated and shown in Figure 1C for AVARH and AVDAT (model $\mathrm{M} 1_{\mathrm{f}}-\mathrm{S}$ ) and in Figure 1D for AVARH and AT1530 (model $\mathrm{M}_{\mathrm{f}^{-}}$ $\mathrm{S})$. For comparisons, the estimated weights from models $\mathrm{M} 1_{\mathrm{f}}$ and $\mathrm{M} 3_{\mathrm{f}}$ (i.e., with no radial smoothing) are also shown in the figures. The estimated temporal-lag parameters and corresponding weights for AVARH were little affected by the addition of a radial smoothing function in the mixed model. The weights for AVARH were very slightly lower at short lags and very slightly higher at long lags for model $\mathrm{M} 1_{\mathrm{f}}-\mathrm{S}$ compared with model $\mathrm{M} 1_{\mathrm{f}}$ (Fig. 1C). The weights for AVARH were even less different between models $\mathrm{M} 3_{\mathrm{f}}-\mathrm{S}$ and $\mathrm{M} 3_{\mathrm{f}}$ (Fig. 1D). Moreover, the estimated marginal effect of AVARH on the response variable $\left(\hat{\beta}^{(1)^{*}}\right)$ was approximately the same whether or not a smoothing function was used (Tables 3 and 5).

Because the polynomial degree was 0 for INTENSITY in the two models being considered, the weights were constant across all time lags by definition and, therefore, are not shown in the figures. However, estimated marginal effect was somewhat lower when the smoothing function was included than when it was not (e.g., $\hat{\beta}^{(3)^{*}}$ was $297 \times 10^{-3}$ for model $\mathrm{M}_{\mathrm{f}}$ [Table 3] and $232 \times 10^{-3}$ for model $\mathrm{M} 1_{\mathrm{f}}-\mathrm{S}$ [Table 5]).
The estimated parameters and the corresponding weights for the temperature-based predictors (AVDAT and AT1530) were substantially affected by the presence of the radial smoothing function in equation 12. When the smoothing function was used, the weights for AVDAT (Fig. 1C) or AT1530 (Fig. 1D) were more negative at both short and very long lags and more positive at intermediate lags compared with the situation when the smoother was not in the model. Furthermore, the estimated marginal effect of either AVDAT or AT1530 $\left(\hat{\beta}^{(2)^{*}}\right)$ was not significantly different from $0(P>0.05)$ (Table 5), based on a $t$ test. In a general sense, the negative effects at the extreme temporal lags cancelled out the positive effects at the intermediate lags, resulting in a null marginal effect of temperature on the response variable.

The standardized marginal effects for AVARH, AVDAT, and INTENSITY for model $\mathrm{M} 1_{\mathrm{f}}-\mathrm{S}$ were estimated as $0.74,0.11$, and 0.61 , respectively. The standardized marginal effects for AVARH, AT1530, and INTENSITY for model $\mathrm{M} 3{ }_{\mathrm{f}}-\mathrm{S}$ were estimated as $0.72,-0.05$, and 0.64 , respectively. These standardized values suggest that AVARH and INTENSITY were of similar importance in predicting $Y$, with a slightly greater impact of AVARH on the response variable. However, the temperature-based predictors were relatively unimportant in predicting $Y$ when a smoothing function was incorporated in the model. The latter result supports the conclusions based on unstandardized marginal effect estimates.

Predictions and model assessments. The predictions of the response variable were very close for $\mathrm{M}_{\mathrm{f}}$ and $\mathrm{M} 3_{\mathrm{f}}$ based on the fit of the full mixed model (equation 9) (Fig. 3A and B). In fact, the correlation coefficient between the two predictions across all observations was $>0.99$. Likewise, for a given model form, the predictions based on the full mixed model $\left(\mathrm{M} 1_{\mathrm{f}}\right.$; equation 9$)$ were very similar to those found based on the composite predictor variables ( $3_{c}$; equation 8). The correlations between these predictions were also $>0.99$. The predictions for models based on the composite variables $\left(\mathrm{M}_{\mathrm{c}}\right.$ and $\left.\mathrm{M} 3_{\mathrm{c}}\right)$ are not shown in Figure 3 because they overlapped to a large extent with the predictions from models $\mathrm{M} 1_{\mathrm{f}}$ and $\mathrm{M} 3_{\mathrm{f}}$. The agreements in predicted log spore densities can be seen by graphing the Studentized residuals from

TABLE 5. Estimated (fixed-effects) parameters for two linear mixed models $\left(\mathrm{M}_{\mathrm{f}}-\mathrm{S}\right.$ and $\mathrm{M} 3_{\mathrm{f}}-\mathrm{S}$; see equation 12$)$ fitted to the log of daily spore density ( $Y$ ) of Gibberella zeae on wheat spikes, where $Y$ on a given day is affected by the predictor (weather) variables on the current $(t)$ and 8 previous days $(t-1 \text { to } t-8)^{\mathrm{a}}$

\begin{tabular}{|c|c|c|c|c|c|c|c|c|}
\hline \multirow[b]{2}{*}{ Time lag } & \multicolumn{4}{|c|}{ Model $\mathrm{M} 1_{\mathrm{f}}-\mathrm{S}^{\mathrm{b}}$} & \multicolumn{4}{|c|}{ Model $\mathrm{M} 3_{\mathrm{f}}-\mathrm{S}^{\mathrm{b}}$} \\
\hline & $\begin{array}{c}\text { AVARH } \\
\left(\times 10^{3}\right)\end{array}$ & $\begin{array}{c}\text { AVDAT } \\
\left(\times 10^{3}\right)\end{array}$ & $\begin{array}{l}\text { INTENSITY } \\
\left(\times 10^{3}\right)\end{array}$ & $\begin{array}{l}\text { Additional } \\
\text { constants }^{\mathrm{c}}\end{array}$ & $\begin{array}{c}\text { AVARH } \\
\left(\times 10^{3}\right)\end{array}$ & $\begin{array}{l}\text { AT1530 } \\
\left(\times 10^{3}\right)\end{array}$ & $\begin{array}{l}\text { INTENSITY } \\
\left(\times 10^{3}\right)\end{array}$ & $\begin{array}{l}\text { Additional } \\
\text { constants }^{c}\end{array}$ \\
\hline$t$ & $10.3(2.8)$ & $-18.2(9.3)$ & $25.8(9.8)$ & $\ldots$ & $10.7(2.7)$ & $-13.2(5.4)$ & $27.3(10.0)$ & $\ldots$ \\
\hline$t-2$ & $8.9(2.1)$ & $5.4(4.8)$ & $25.8(9.8)$ & $\ldots$ & $9.0(2.1)$ & $0.6(3.3)$ & $27.3(10.0)$ & $\ldots$ \\
\hline$t-3$ & $8.3(1.9)$ & $11.9(5.0)$ & $25.8(9.8)$ & $\ldots$ & $8.2(1.9)$ & $4.4(3.4)$ & $27.3(10.0)$ & $\ldots$ \\
\hline$t-4$ & $7.6(1.8)$ & $14.7(5.3)$ & $25.8(9.8)$ & $\ldots$ & $7.3(1.8)$ & $6.1(3.5)$ & $27.3(10.0)$ & $\ldots$ \\
\hline$t-8$ & $4.9(2.6)$ & $-10.0(8.6)$ & $25.8(9.8)$ & $\ldots$ & $4.0(2.5)$ & $-8.2(5.0)$ & $27.3(10.0)$ & $\ldots$ \\
\hline Marginal $^{\mathrm{d}}$ & $68.3(16.2)$ & $24.2(33.9)$ & $232.3(88.2)$ & & $66.2(16.2)$ & $-8.2(23.7)$ & $245.9(90.3)$ & \\
\hline Intercept & $\ldots$ & $\ldots$ & $\ldots$ & $-0.6(1.6)$ & $\ldots$ & .. & $\ldots$ & $0.3(1.3)$ \\
\hline \multicolumn{9}{|l|}{ Location } \\
\hline Indiana & $\ldots$ & $\ldots$ & $\ldots$ & $-2.5(0.7)$ & $\ldots$ & $\ldots$ & $\ldots$ & $-2.6(0.7)$ \\
\hline Manitoba & $\ldots$ & $\ldots$ & $\ldots$ & $-1.5(1.1)$ & $\ldots$ & $\ldots$ & $\ldots$ & $-1.5(1.1)$ \\
\hline
\end{tabular}

${ }^{\text {a }}$ Parameter estimates for the predictor variables are constrained to follow a polynomial of order of $d=2$ for average daytime air temperature $\left({ }^{\circ} \mathrm{C}\right)(\mathrm{AVDAT})$ and number of hours per day with air temperature of 15 to $30^{\circ} \mathrm{C}$ (AT1530), $d=1$ for average daily relative humidity of the air (AVARH), or $d=0$ for daily average rainfall intensity (INTENSITY). See text for description of the variables.

${ }^{\mathrm{b}}$ Standard errors in parentheses. Estimated parameters and standard errors for weather predictors (for time lags of $t$ to $t-8$ and the marginal effects) multiplied by 1,000 for ease of presentation.

${ }^{c}$ Estimates of $\alpha$ (intercept) and the $L_{i}$ location effects in equation 12. All effects are on a log scale.

${ }^{\mathrm{d}}$ Marginal parameter estimate in a given column is the sum of the individual estimates listed for the 9 days. Because of rounding in the presentation of the individual estimates, the sum of the shown time-lag coefficients may be slightly different from the presented estimated marginal effect (at the last decimal place). 
these models in a matrix plot (Fig. 4). These model predictions agreed, overall, in terms of the magnitude and direction of the errors.

The predicted log spore densities based on the full mixed models with a radial smoother are also shown in Figs. 3A (model $\mathrm{M} 1_{\mathrm{f}}-\mathrm{S}$ ) and $3 \mathrm{~B}$ (model $\mathrm{M} 3_{\mathrm{f}}-\mathrm{S}$ ). These predictions were also similar to those found for models without the smoother, although slight differences can be seen. As shown by the CCC values (or AICC), the use of the smoother resulted in some improvement in fit (Table 4). The correlation of the predictions for models $\mathrm{M} 1_{\mathrm{f}}-\mathrm{S}$ and $\mathrm{M} 3_{\mathrm{f}}-\mathrm{S}$ was $>0.99$. The agreement can also be seen by the graph of the Studentized residuals for $\mathrm{M}_{\mathrm{f}}-\mathrm{S}$ and $\mathrm{M} 3_{\mathrm{f}}-\mathrm{S}$ (Fig. 4). The matrix plot also shows that there was somewhat more variation in the predictions for models with and without the smoothing function (e.g., $\mathrm{M} 1_{\mathrm{f}}$ versus $\mathrm{M} 1_{\mathrm{f}}-\mathrm{S}$ ).

Plots of Studentized residuals versus predicted values for any single model (of the best ones described here $\left[\mathrm{M} 1_{\mathrm{f}}, \mathrm{M} 3_{\mathrm{f}}, \mathrm{M} 1_{\mathrm{f}}-\mathrm{S}\right.$, and $\left.\mathrm{M} 3_{\mathrm{f}}-\mathrm{S}\right]$ ) showed a random scatter; moreover, the normal probability plot revealed a straight line for each of these models (L. V. Madden, unpublished). This indicates that these final models were reasonable representations of the profiles of $\log$ spore density, and that there was no evidence of a nonlinear relationship between $Y$ and the predictors.

\section{DISCUSSION}

There is a need in many disciplines to quantify the relationship between a time-varying response variable and one or more timevarying predictor variables $(4,6,23-25,34,39-41,43,48)$, either to understand biological or physical processes or to make predictions of the response at future times. Quantification and modeling are hampered, however, by the serial correlation of the predictors and the response variable over time $(20,29,37,38,48)$. For instance, in situations where the effect of a predictor is manifested over several days, estimated parameters of models such as equation $6 a$ will be very imprecise with large standard errors if unconstrained ordinary least squares or ML is used $(17,20,47)$. This leads to a wide scatter of parameter estimates for the different lagged predictor variables and an unstable model fit. Thus, various methods have been developed to correct for, or account for, the correlations $(3,11,39,40,46-48)$. In a sense, the various approaches smooth out the excess variation in estimated parameters compared with the usual model fits, typically by constraining the parameter estimates in some implicit or explicit manner or by smoothing the predictors or response variables using some form of parametric or nonparametric (smoothing) function. PDL regression is one approach to the problem which is popular in econometrics $(4,17,20)$ and has been used by several investigators to quantify time-dependent processes $(23,39,43,44)$.

Most applications of PDL and other constrained-parameter models involve the analysis of single (long) profiles of data and, where appropriate, the comparison of the parameter estimates from separate data sets, such as for human mortality in different cities in response to certain types of air pollution $(6,11,34$, $35,40,46)$. Parameter estimates from separate data sets (separate model fits) are then sometimes combined using meta-analytical methods $(5,6,46)$. In plant pathology, long profiles of observations (e.g., consisting of $\geq 100$ consecutive days of data) are not common, preventing the direct utilization of some of the developed statistical methods for time-varying response variables. The

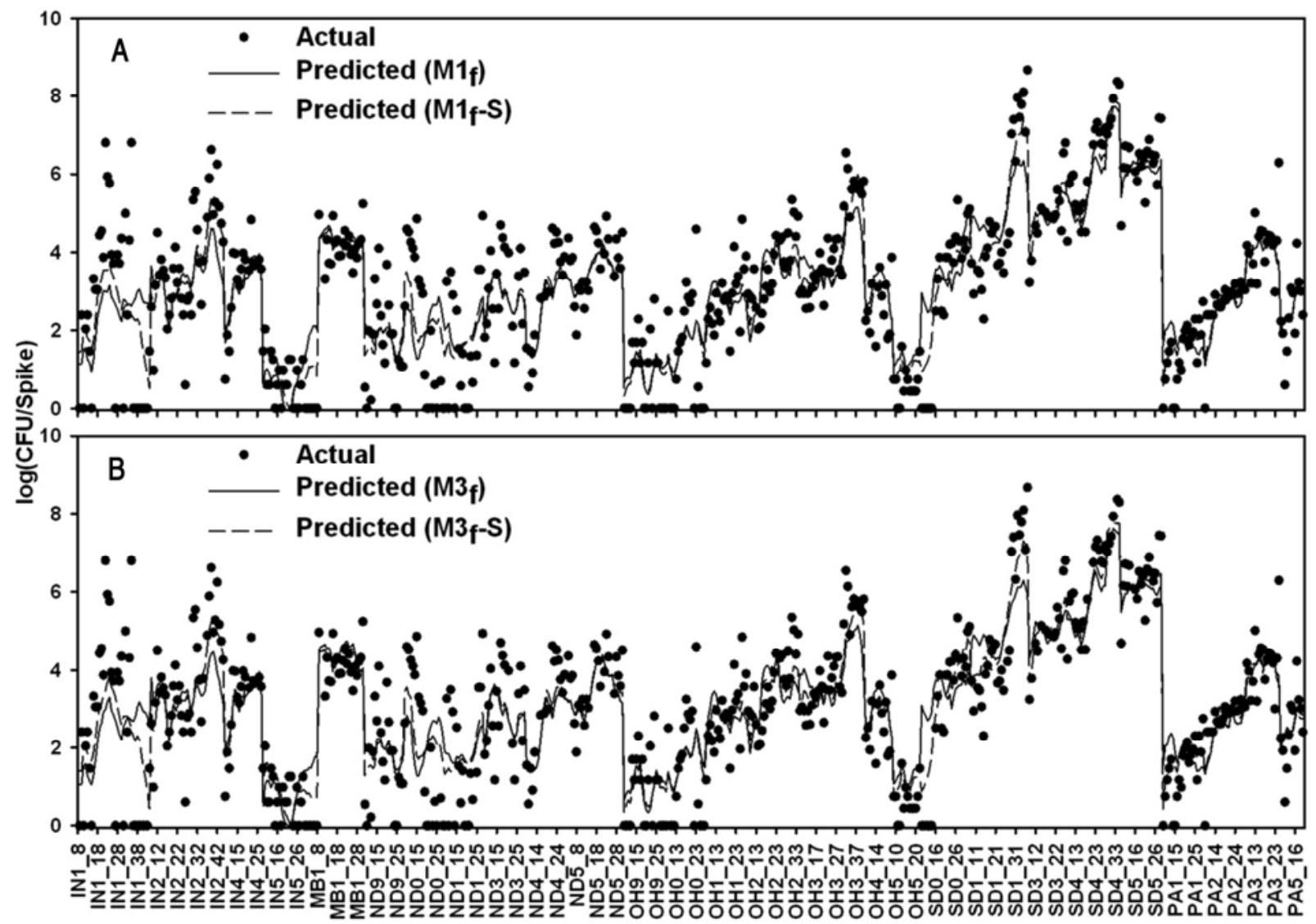

Fig. 3. Observed and predicted log of daily spore density of Gibberella zeae on wheat spikes across all location-years and times, based on the fit of linear mixed models to the data. A, Fit of model $\mathrm{M} 1_{\mathrm{f}}$ (equation 9) and $\mathrm{M} 1_{\mathrm{f}}-\mathrm{S}$ (equation 12) to the data, based on the following predictors: average daily relative humidity of the air (AVARH), average daytime air temperature $\left({ }^{\circ} \mathrm{C}\right)$, and daily average rainfall intensity $(\mathrm{mm} / \mathrm{h})\left(\right.$ INTENSITY). B, Fit of model $\mathrm{M}_{\mathrm{f}}(\mathrm{equation} 9)$ and $\mathrm{M} 3_{\mathrm{f}}-\mathrm{S}$ (equation 12) to the data, based on the following predictors: AVARH, number of hours per day with air temperature of 15 to $30^{\circ} \mathrm{C}$, and INTENSITY. Order of the locations is arbitrary. Only a small fraction of the individual time values are labeled on the horizontal axis to avoid excessive crowding of text. 
challenges in data analysis are clearly demonstrated with the FHB example used in this article (33). Although the profiles of G. zeae inoculum density in wheat fields encompassed the relevant period of spore production and plant infection for this pathosystem $(10,14)$, the profile duration was insufficient to estimate the PDL parameters for any single location-year (33). However, by pooling data across groups (i.e., location-years), Paul et al. (33) showed that it was possible to successfully fit PDL-type mixed models in two stages to the inoculum-density profiles, and account for group effects (e.g., overall inoculum levels or specific methods for inoculum quantification), serial correlation of residuals, and the serial and cross-correlation of the compositevariable predictors (equation 8). Because the weights given to each time lag of each predictor in determining composite variables cannot vary in the second stage of this analysis, we undertook the current investigation to explore a less restrictive mixed modeling approach for the data analysis. We have shown here that it is computationally possible to incorporate distributed-lag constraints on the parameters in a full linear mixed model using the GLIMMIX (or MIXED) procedures in SAS. The approach is based on explicit calculation of $Z_{(k)}$ variables, which do not involve unknown parameters, for any lag length $(n)$ and for each of the relevant polynomial orders $(d)$.
The ML-based approach taken in this investigation for model fitting allowed us to utilize an information-theoretic approach to evaluate the relative fit of one model to another. Specifically, using the methods advocated by Burnham and Anderson $(7,8)$, based on the so-called corrected AICC statistic for each fitted model, we could clearly show which models (of the ones considered here as being of potential value) had the highest probability of being the best. Although not required, this approach can also be viewed as a Bayesian procedure for selecting the best model, with certain prior probabilities placed on the population (set) of models under consideration (8). The full linear mixed model versions of models 1 and $3\left(\mathrm{M}_{\mathrm{f}}\right.$ and $\mathrm{M} 3_{\mathrm{f}}$, both based on the use of three weather variables) were clearly the best choices of all the models without a smoothing function using the AICC criterion.

Results from the fit of the full linear mixed model (equation 9) were quite similar to those found with the more restrictive mixed model with composite predictors (equation 8; Figs. 1 and 4). For the best-fitting models $\left(\mathrm{M}_{\mathrm{f}}\right.$ and $\left.\mathrm{M} 3_{\mathrm{f}}\right)$, predictions of $Y$ were virtually indistinguishable and the estimated marginal effects for the predictors were also similar for the two modeling approaches. Moreover, for the predictor with a first-order polynomial constraint (AVARH), the weights estimated with the full mixed model

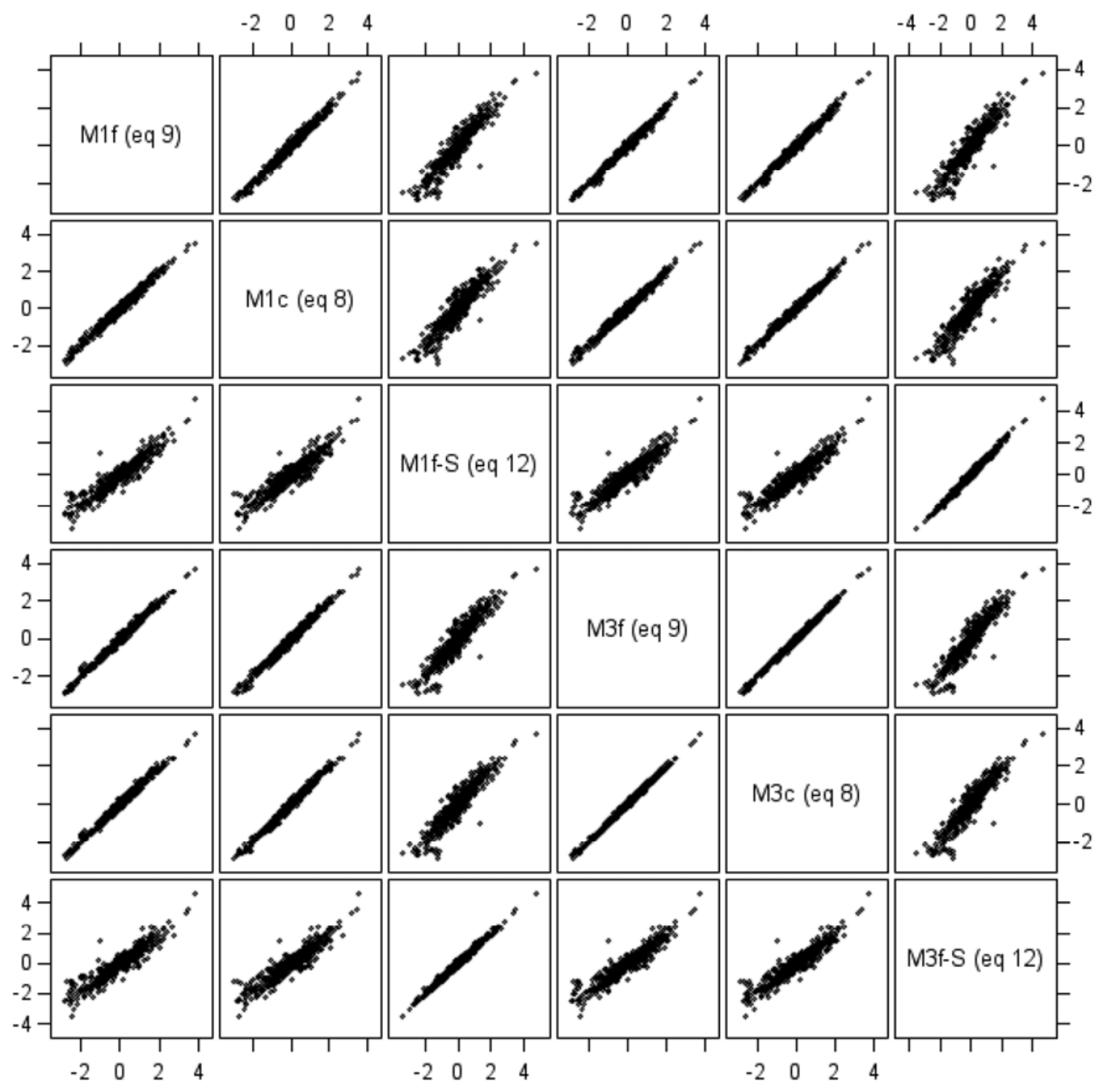

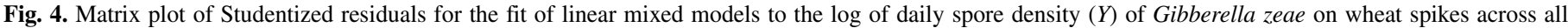

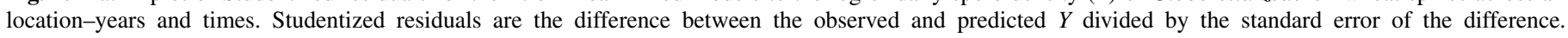
Studentized residuals are shown for the fit of model $\mathrm{M} 1_{\mathrm{f}}$, model M1 $1_{\mathrm{c}}$, model $\mathrm{M} 1_{\mathrm{f}}-\mathrm{S}$, model M $3_{\mathrm{f}}$, model M $3_{\mathrm{c}}$, and model M $3_{\mathrm{f}}-\mathrm{S}$. 
fit were very close to those used previously in constructing the composite variable (Fig. 1A and B). The similarity was also found for other predictors with first-order constraints in models not reported here (L. V. Madden, unpublished). The largest discrepancy in weights was for predictors with second-degree constraints (e.g., AVDAT $\left[\mathrm{M} 1_{\mathrm{f}}\right.$ versus $\left.\mathrm{M} 1_{\mathrm{c}}\right]$ and AT1530 [M3 ${ }_{\mathrm{f}}$ versus $\left.\mathrm{M} 3_{\mathrm{c}}\right]$ ); the largest discrepancies in estimated marginal effects were also for the AVDAT predictor. It is not known whether discrepancy in the weights for the two modeling approaches will increase for other data sets, in general, as the order of the polynomial constraint increases.

For single long profiles of observations, it is common to use spline or LOESS smoothing functions to remove long-term (or seasonal) variation in the response variable that is probably not related to the direct effects of the predictor variables of interest $(11,39,40,46)$. This is directly done within the framework of generalized additive models (GAMs) $(36,39)$, available in many statistical software packages (e.g., GAM procedure in SAS). Choosing the degree of smoothing with GAM models is challenging with data collected over time; the commonly used generalized cross validation method for $\lambda$ estimation typically results with an overly rough fit when the data are serially correlated. Thus, most investigators simply choose a $d f_{S}$ value a priori that generally corresponds to a desired level of smoothing (39). GAMs in their standard usage also do not accommodate multiple random effects and complex serial correlations of residuals, which makes them unsuitable for our purposes. However, penalized and other types of smoothing splines can also be fitted using mixed-model methods, as pointed out by Speed (42) and others $(18,28,31,45)$; thus, smooth functions (e.g., $S_{i j}\left(t_{\mathrm{t}}\right)$ in equation 12) can be included in a wide class of mixed models, such as those used in our investigation, to determine the appropriate degree of smoothness for a given data set while simultaneously estimating the distributed-lag parameters for the predictor variables.

The fit of equation 12 to the example data without weather predictors indicated that there was, in general, a very smooth temporal trend (i.e., with a small $d f_{S}$ ) in $\log$ spore density, with a slight upward trend in several location-years. Incorporation of the smoothing function into the mixed models with weather predictors $\left(\mathrm{M}_{\mathrm{f}}-\mathrm{S}\right.$ and $\mathrm{M} 3_{\mathrm{f}}-\mathrm{S}$; equation 12) did improve the fit to the data compared with the situation without a smoothing function. In part, this is because the smoothing component accounted for the (small) overall temporal trends that would not be captured by the simpler equation 9 . Interestingly, most of the parameter estimates were not greatly affected by the inclusion of the smoothing function. The estimated weights (or individual $\beta_{m}$ estimates) and marginal effect for AVARH were very similar between the pairs of models (with and without the smoother) (Fig. 1C and D). The estimated effects of INTENSITY were diminished somewhat in the models with the smoothing function, although the predictor effect was still highly significant. Incorporation of the smoothing function had the greatest impact on the estimated effects of the temperature variables (AVDAT in $\mathrm{M}_{\mathrm{f}}-\mathrm{S}$ and AT1530 in $\mathrm{M}_{\mathrm{f}}-\mathrm{S}$ ), which were the predictors with a seconddegree polynomial constraint. The weights were more variable over time, and the marginal effects were considerably smaller and no longer significant for these temperature predictors. This suggests that the general trend in $Y$ over time (identified through the smoothing function) was mostly due to trends in the ambient temperature, with deviations from the overall trends being influenced by rain intensity and, especially, relative humidity. However, it is also possible that the general trends in $Y$ were also due to some other (unmeasured) variables that were highly correlated with AVDAT and AT1530. It is quite possible with other systems, of course, that the general trends over time will not be redundant with any of the predictor variables in the models. Moreover, it should be noted that a smoothing function can also be added to mixed models using composite weather variables (i.e., adding $S_{i j}\left(t_{t}\right)$ to equation 8 ), although we did not explore this approach here.

In conclusion, we have shown that it is possible to represent highly variable and serially correlated sequences of biological data-such as log of spore density—-from multiple locations and years using a full linear mixed model with polynomialdistributed-lag constraints on the fixed-effect parameter estimates. The approach can be adapted for modeling profiles of any response data where there are multiple sources of variation and correlated observations; thus, the methodology has great potential for dealing with time-varying profiles of spore density or vector abundance. Because the approach is based on mixed-model theory, smoothing functions can be directly incorporated to account for unspecified trends in the data. Interestingly, and importantly, results obtained here were very similar to those found previously using a two-step protocol, where the time-lag weights were found in the first step and then the marginal effects (and other random effect terms) were estimated in the second step. Thus, with this example data set, modeling results were robust to the statistical methodology utilized, and all the general conclusions made by Paul et al. (33) still hold based on the current analysis.

Analyses of additional data sets will be required to determine how often the results will be in general agreement for the oneand two-stage approaches. Use of two-stage approach is definitely more convenient and practical, because utilization of the full mixed model (equation 9 or 12) demands much more of the investigator. For instance, $Z_{(k)}$ variables need to be calculated for each predictor in a full mixed model, where the number of terms (and hence the coefficients) are different for each maximum time lag; moreover, the number of $\mathrm{Z}_{(k)}$ variables (for each predictor) depends on the polynomial-constraint order that is being considered. Selecting the predictor variables out of a large pool of candidate predictors, together with the maximum time lag and polynomial order for each, will be a very tedious undertaking, although macros could be written to streamline the process. Also, with a sufficiently large number of variables, optimization of the ML-estimation procedure could be hindered. PDL regression procedures (e.g., PDLREG procedure in SAS), although not fully accounting for all the relevant components of the experimental design, make it quite easy to evaluate individual predictors for their potential (or approximate) effect on the response variable, where the effect is distributed over several days. Thus, we recommend that PDL regression methods be used as component of any data-mining exercise for these situations; the preliminary PDL results can then be used to either directly construct composite predictors or guide the choice of $Z_{(k)}$ variables in a full mixed model (as done here).

\section{APPENDIX}

Polynomial distributed lags. The derivation of a polynomial distributed lag model can be demonstrated for a polynomial of degree $d=2$ (quadratic pattern to the parameters). Starting with the model in equation 1a for a relationship between $Y_{t}$ and $X_{t-m}$ $(m=0, \ldots, n)$, with the constraints on the $\beta_{m}$ parameters given by equation 2 (using $d=2$ ), one can write the model as:

$$
\begin{aligned}
Y_{t}= & \alpha+\eta_{0} X_{t}+\left(\eta_{0}+\eta_{1}+\eta_{2}\right) X_{t-1}+\left(\eta_{0}+2 \eta_{1}+4 \eta_{2}\right) X_{t-2}+ \\
& \ldots+\left(\eta_{0}+m \eta_{1}+m^{2} \eta_{2}\right) X_{t-m}+\cdots+\left(\eta_{0}+n \eta_{1}+n^{2} \eta_{2}\right) X_{t-n}+e_{t}
\end{aligned}
$$

Algebraic rearrangement of equation A1 results in:

$$
\begin{aligned}
Y_{t}= & \alpha+\eta_{0}\left(X_{t}+X_{t-1}+X_{t-2}+\ldots+X_{t-m}+\cdots+X_{t-n}\right)+ \\
& \eta_{1}\left(0 X_{t}+1 X_{t-1}+2 X_{t-2}+\ldots+m X_{t-m}+\cdots+n X_{t-n}\right)+ \\
& \eta_{2}\left(0 X_{t}+1 X_{t-1}+4 X_{t-2}+\ldots+m^{2} X_{t-m} \cdots+n^{2} X_{t-n}\right)+e_{t}
\end{aligned}
$$


Equation A2 can be written equivalently as:

$$
Y_{t}=\alpha+\eta_{0} \sum_{m=0}^{n} X_{t-m}+\eta_{1} \sum_{m=0}^{n} m X_{t-m}+\eta_{2} \sum_{m=0}^{n} m^{2} X_{t-m}+e_{t}
$$

The terms in the parentheses can be written, respectively, as:

$$
\begin{gathered}
Z_{(0) t}=\sum_{m=0}^{n} X_{t-m} \\
Z_{(1) t}=\sum_{m=0}^{n} m X_{t-m} \\
Z_{(2) t}=\sum_{m=0}^{n} m^{2} X_{t-m}
\end{gathered}
$$

which do not involve unknown parameters. Equation A2 can thus be written succinctly as a linear model with three predictor variables (with $d=2$ ):

$$
Y_{t}=\alpha+\eta_{0} Z_{(0) t}+\eta_{1} Z_{(1) t}+\eta_{2} Z_{(2) t}+e_{t}
$$

More generally, we can define the variable $Z_{(k) t}$ as:

$$
Z_{(k) t}=\sum_{m=0}^{n} m^{k} X_{t-m}
$$

and write equation A3 even more succinctly as:

$$
Y_{t}=\alpha+\sum_{k=0}^{d} \eta_{k} Z_{(k) t}+e_{t}
$$

Polynomials of any order can be specified by the choice of $k$ in equation of A4.

\section{ACKNOWLEDGMENTS}

Salaries and research support were provided by state and federal funds to the Ohio Agricultural Research and Development Center. This investigation is based upon work supported, in part, by the United States Department of Agriculture (USDA) (agreement no. 59-0790-4-112). Further support was supplied by USDA-Cooperative State Research, Education and Extension Service Special Grant 2008-34493-19444. This is a cooperative project with the U.S. Wheat \& Barley Scab Initiative. Any opinions, findings, conclusions, or recommendations expressed in this publication are those of the authors and do not necessarily reflect the view of the USDA. We thank P. E. Lipps, E. De Wolf, G. Shaner, G. Buechley, T. Adhikari, S. Ali, J. Stein, and L. Osborne for their cooperation and collaboration in conducting the studies.

\section{LITERATURE CITED}

1. Almon, S. 1965. The distributed lag between capital appropriations and expenditures. Econometrica 33:178-196.

2. Angelosante Bruno, A., Pace, L., Tomassetti, B., Coppola, E., Verdecchia, M., Pacioni, G., and Visconti, G. 2007. Estimation of fungal spore concentrations associated to meteorological variables. Aerobiologia 23:221-228.

3. Armstrong, B. 2006. Models for the relationship between ambient temperature and daily mortality. Epidemiology 17:624-631.

4. Batten, D. S., and Thornton, D. L. 1983. Polynomial distributed lags and the estimation of the St. Louis equation. Federal Reserve Bank of St. Louis Review April:13-25.

5. Berhane, K., and Thomas, D. C. 2002. A two-stage model for multiple time series data of counts. Biostatistics 3:21-32.

6. Braga, A. L. F., Zanobetti, A., and Schwartz, J. 2001. The time course of weather-related deaths. Epidemiology 12:662-667.

7. Burnham, K. P., and Anderson, D. R. 2002. Model Selection and Multimodel Inference: A Practical Information-Theoretical Approach, 2nd ed. Springer, New York.

8. Burnham, K. P., and Anderson, D. R. 2004. Multimodel inference: understanding AIC and BIC in model selection. Sociol. Methods Res. 33:261-304.

9. Crisafulli, D., Almqvist, C., Marks, G., and Tovey, E. 2007. Seasonal trends in house dust mite allergen in children's beds over a 7-year period. Allergy 62:1394-1400.
10. De Wolf, E., Francl, L., Lipps, P., Madden, L., Osborne, L., and Jin, Y. 2000. Factors affecting the development of wheat Fusarium head blight. Pages 137-140 in: Proc. 2000 Natl. Fusarium Head Blight Forum, Erlanger, KY.

11. Dominici, F., McDermott, A., Zeger, S., and Samet, J. M. 2003. Airborne particulate matter and mortality: timescale effects in four US cities. Am. J. Epidemiol. 157:1055-1065.

12. Edwards, L. J., Stewart, P. W., Muller, K. E., and Helms R. W. 2001. Linear equality constraints in the general linear mixed model. Biometrics 57:1185-1190.

13. Fitt, B. D. L., and McCartney, H. A. 1986. Spore dispersal in relation to epidemic models. Pages 311-345 in: Plant Disease Epidemiology: Population Dynamics and Management. K. J. Leonard and W. E. Fry, eds. Macmillan Publishers, New York.

14. Francl, L., Shaner, G., Bergstrom, G., Gilbert, J., Pedersen, W., DillMacky, R., Sweets, L., Corwin, B., Jin, Y., Gallenberg, D., and Wiersma, J. 1999. Daily inoculum levels of Gibberella zeae on wheat spikes. Plant Dis. 83:662-666.

15. Ganguli, B., and Wand, M. P. 2007. Feature significance in generalized additive models. Stat. Comput. 17:179-192.

16. Grinn-Gofron, A, and Mika, A. 2008. Selected airborne allergenic fungal spores and meteorological factors in Szczecin, Poland, 2004-2006. Aerobiologia 24:89-97.

17. Gujarati, D. N., and Porter, D. C. 2009. Basic Econometrics, 5th ed. McGraw-Hill, New York.

18. Gurrin, L. C., Scurrah, K. J., and Hazelton, M. L. 2005. Tutorial in biostatistics: spline smoothing with linear mixed models. Stat. Med. 24:3361-3381.

19. Hastie, T. J., and Tibshirani, R. J. 1990. Generalized Additive Models. Chapman \& Hall, New York.

20. Hill, R. C., Griffiths, W. E., and Judge, G. G. 1997. Undergraduate Econometrics. John Wiley \& Sons, New York.

21. Hsiao, C. 2003. Analysis of Panel Data. Cambridge University Press, UK.

22. Huang,Y. J., Fitt, B. D. L., Jedryczka, M., Dakowska, S., West, J., Gladders, P., Steed, J. M., and Li, Z.-Q. 2005. Patterns of ascospore release in relation to Phoma stem canker epidemiology in England (Leptosphaeria maculans) and Poland (Leptosphaeria biglobosa). Eur. J. Plant Pathol. 111:263-277.

23. Laranjeira, F. F., Bergamin Filho, A., Amorim, L., and Lopes, J. R. S. 2003. Comportamento sazonal da clorose variegada dos citros em três regiões do Estado de São Paulo. Fitopatol. Bras. 28:633-641.

24. Le Tertre, A., Schwartz, J., and Touloumi, G. 2005. Empirical Bayes and adjusted estimates approach to estimating the relation of mortality to exposure of $\mathrm{PM}_{10}$. Risk Anal. 25:711-718.

25. Lima, M. A., Maffia, L. A., Barreto, R. W., and Mizubuti, E. S. G. 2009. Phytophthora infestans in a subtropical region: survival on tomato debris, temporal dynamics of airborne sporangia and alternative hosts. Plant Pathol. 58:87-99.

26. Littell, R. C., Milliken, G. A., Stroup, W. W., Wolfinger, R. D., and Schabenberger, O. 2006. SAS System for Mixed Models. SAS Institute, Cary, NC.

27. Liu, H., Zheng, Y., and Shen, J. 2008. Goodness-of-fit measures of $R^{2}$ for repeated measures mixed effect models. J. Appl. Stat. 35:1081-1092.

28. Madden, L. V. 2009. Analysis of temporal observations in epidemics when there is no definable functional relationship between the variable and time. Pages 86-87 in: Proc. 10th Int. Epidemiol. Workshop. D. M. Gadoury, R. C. Seem, M. Moyer, and W. E. Fry, eds. Cornell University, New York.

29. Madden, L. V., Hughes, G., and van den Bosch, F. 2007. The Study of Plant Disease Epidemics. American Phytopathological Society Press, St. Paul, MN.

30. Morton, R., Kang, E. L., and Henderson, B. L. 2009. Smoothing splines for trend estimation and prediction in time series. Environmetrics 20:249259.

31. Ngo, L., and Wand, M. P. 2004. Smoothing with mixed model software. J. Stat. Software 9:1-54 (online journal).

32. Pagano, M., and Hartley, M. J. 1981. On fitting distributed lag models subject to polynomial restrictions. J. Econometrics 16:171-198.

33. Paul, P. A., Lipps, P. E., De Wolf, E., Shaner, G., Buechley, G., Adhikari, T., Ali, S., Stein, J., Osborne, L., and Madden, L. V. 2007. A distributedlag analysis of the relationship between Gibberella zeae inoculum density on wheat spikes and weather variables. Phytopathology 97:1608-1624.

34. Peng, R. D., Dominici, F., and Louis, T. A. 2006. Model choice in time series studies of air pollution and mortality. J. R. Stat. Soc. A 169 (part 2):179-203.

35. Post, E., Hoaglin, D., Deck, L., and Larntz, K. 2001. An empirical Bayes approach to estimating the relation of mortality to exposure to particulate matter. Risk Anal. 21:837-842.

36. Ruppert, D., Wand, M. P., and Carroll, R. J. 2003. Semiparametric Regression. Cambridge University Press, Cambridge. 
37. Schabenberger, O, and Gotway, C. A. 2005. Statistical Methods for Spatial Data Analysis. Chapman \& Hall/CRC, Boca Raton, FL.

38. Schabenberger, O., and Pierce, F. J. 2002. Contemporary Statistical Models for the Plant and Soil Sciences. CRC Press, New York.

39. Schwartz, J. 2000. The distributed lag between air pollution and daily deaths. Epidemiology 11:320-326.

40. Schwartz, J., Laden, F., and Zanobetti, A. 2002. The concentrationresponse relation between PM2.5 and daily deaths. Environ. Health Perspect. 110:1025-1029.

41. Sims, M., Elston, D. A., Larkham, A., Nussey, D. H., and Albon, S. D. 2007. Identifying when weather influences life-history traits of grazing herbivores. J. Anim. Ecol. 76:761-770.

42. Speed, T. 1991. Comment. Stat. Sci. 6:42-44.

43. Teklehaimanot, H. D., Lipsitch, M., Teklehaimanot, A., and Schwartz, J. 2004. Weather-based prediction of Plasmodium falciparum malaria in epidemic-prone regions of Ethiopia I. Patterns of lagged weather effects reflect biological mechanisms. Malaria J. 41:1-11.
44. Van Hemelrijck, M. J. J., Lindblade, K. A., Kubaje, A., Hamel, M. J., Odhiambo, F., Phillips-Howard, P. A., Laserson, K. F., Slutsker, L., and Feikin, D. R. 2009. Trends observed during a decade of paediatric sick visits to peripheral health facilities in rural western Kenya, 1997-2006. Trop. Med. Int. Health 14:62-69.

45. Welham, S. J., Cullis, B. R., Kenward, M. G., and Thompson, R. 2007. A comparison of mixed model splines for curve fitting. Aust. N. Z. J. Stat. 49:1-23.

46. Zanobetti, A., Schwartz, J., Samoli, E., Gryparis, A., Touloumi, G., Atkinson, R., Le Tertre, A, Bobros, J., Celko, M., Goren, A., Forsberg, B., Michelozzi, P., Rabczenko, D., Aranguez Ruiz, E., and Katsouyanni, K. 2002. Epidemiology 13:87-93.

47. Zanobetti, A., Wand, M. P., Schwartz, J., and Ryan, L. M. 2000 Generalized additive distributed lag models: quantifying mortality displacement. Biostatistics 1:279-292.

48. Zeger, S. L., Irizarry, R., and Peng, R. D. 2006. On time series analysis of public health and biomedical data. Annu. Rev. Public Health 27:57-79. 